\title{
Existence analysis of a degenerate diffusion system for heat-conducting gases
}

\author{
Gianluca Favre@, Ansgar Jüngel[1, Christian Schmeiser and \\ Nicola Zamponi
}

\begin{abstract}
The existence of global weak solutions to a parabolic energytransport system in a bounded domain with no-flux boundary conditions is proved. The model can be derived in the diffusion limit from a kinetic equation with a linear collision operator involving a non-isothermal Maxwellian. The evolution of the local temperature is governed by a heat equation with a source term that depends on the energy of the distribution function. The limiting model consists of cross-diffusion equations with an entropy structure. The main difficulty is the nonstandard degeneracy, i.e., ellipticity is lost when the gas density or temperature vanishes. The existence proof is based on a priori estimates coming from the entropy inequality and the $H^{-1}$ method and on techniques from mathematical fluid dynamics (renormalized formulation, div-curl lemma).
\end{abstract}

Mathematics Subject Classification. 35K51, 35K65, 35Q79, 76R50, 82C40.

Keywords. Cross-diffusion, Energy transport, Weak solutions, Renormalized equation, Compensated compactness.

\section{Introduction}

This paper is concerned with the global existence analysis of a degenerate diffusion system governing the evolution of the particle density $\rho(x, t)$ and temperature $\theta(x, t)$ :

$$
\partial_{t} \rho=\Delta(\rho \theta), \quad \partial_{t} E=\Delta\left(\theta+\frac{5}{2} \rho \theta^{2}\right) \quad \text { in } \Omega, t>0,
$$

The first three authors acknowledge partial support from the FWF, the Austrian Science Fund (FWF), grants F65 and W1245. The second author has been additionally supported by the grants P30000 and P33010 of the FWF. The fourth author acknowledges support from the Alexander von Humboldt Foundation. 
where $E=\theta+\frac{3}{2} \rho \theta$ is the energy density, supplemented by no-flux boundary and initial conditions,

$$
\begin{gathered}
\nabla(\rho \theta) \cdot \nu=\nabla\left(\theta+\frac{5}{2} \rho \theta^{2}\right) \cdot \nu=0 \quad \text { on } \partial \Omega, t>0, \\
\rho(0)=\rho^{0}, E(0)=E^{0}:=\theta^{0}+\frac{3}{2} \rho^{0} \theta^{0} \quad \text { in } \Omega,
\end{gathered}
$$

and $\Omega \subset \mathbb{R}^{3}$ is a bounded domain. The equations describe a rarefied gas that exchanges heat with the background, coupled through the energy exchange. They can be formally derived from a collisional kinetic equation, coupled to a heat equation for the background temperature governed by a Fourier law, and they are written in dimensionless form. We refer to Sect. 2 for modeling details.

A major difficulty of system (1) is the derivation of suitable a priori estimates. This issue will be tackled by exploiting the entropy structure of the system. This means that Eq. (1) can be written in the cross-diffusion form

$$
\partial_{t} \vec{u}=\operatorname{div}(B \nabla \vec{q})
$$

where

$$
\vec{u}=\left(\begin{array}{c}
\rho \\
E
\end{array}\right), \quad \vec{q}=\left(\begin{array}{c}
\log \left(\rho / \theta^{3 / 2}\right)+\frac{5}{2} \\
-1 / \theta
\end{array}\right), \quad B=\left(\begin{array}{cc}
\rho \theta & \frac{5}{2} \rho \theta^{2} \\
\frac{5}{2} \rho \theta^{2} & \theta^{2}\left(1+\frac{35}{4} \rho \theta\right)
\end{array}\right) .
$$

The so-called Onsager matrix $B$ is symmetric and positive semidefinite. However, $B$ becomes indefinite when $\rho=0$ or $\theta=0$, showing that (4) is of degenerate type. The Gibbs free energy

$$
G=\rho \theta \log \frac{\rho}{\theta^{3 / 2}}+\frac{3}{2} \rho \theta-\theta(\log \theta-1),
$$

defines the

- chemical potential $\mu=\partial G / \partial \rho=\theta\left(\log \left(\rho / \theta^{3 / 2}\right)+\frac{5}{2}\right)$,

- the (mathematical) entropy $h=\partial G / \partial \theta=\rho \log \left(\rho / \theta^{3 / 2}\right)-\log \theta$, and

- the energy density $E=G-\theta \partial G / \partial \theta=\left(1+\frac{3}{2} \rho\right) \theta$.

We reveal the formal gradient-flow structure for (4) by defining the thermochemical potential $\phi=\partial h / \partial \rho=\mu / \theta$ and the negative inverse temperature $\partial h / \partial E=-1 / \theta$ (interpreting $h$ as a function of $(\rho, E))$ such that

$$
\partial_{t}(\rho, E)^{T}-\operatorname{div}(B \nabla \mathrm{D} h)=0
$$

where $\mathrm{D} h$ is the vector with components $\partial h / \partial \rho$ and $\partial h / \partial E$. Furthermore, the entropy $h$ is a Lyapunov functional along solutions to (4):

$$
\frac{d}{d t} \int_{\Omega} h d x=\int_{\Omega}\left(\frac{\partial h}{\partial \rho} \partial_{t} \rho+\frac{\partial h}{\partial E} \partial_{t} E\right) d x=-\int_{\Omega} \nabla(\mathrm{D} h)^{T} B \nabla \mathrm{D} h d x \leq 0
$$

since $M$ is positive semidefinite. In particular, we obtain a priori estimates for $\nabla(\mathrm{D} h)^{T} B \times \nabla \mathrm{D} h$ in $L^{1}(\Omega)$, from which we conclude gradient estimates for $\sqrt{\rho \theta}$ and $\log \theta$ in $L^{2}(\Omega)$ (see below).

Still, this approach is not sufficient. Indeed, because of the degeneracy at $\theta=0$, we cannot expect to achieve any control on the gradient of $\rho$, and moreover, the bounds from the entropy estimate are not sufficient to conclude. 
Our idea, detailed below, is to apply well-known tools from mathematical fluid dynamics like $H^{-1}$ estimates and compensated compactness. The originality of this work consists in the combination of these tools and entropy methods, which allows us to treat non-standard degeneracies. We remark that degenerate mobilities were also treated in Cahn-Hilliard equations; see, e.g., [1,11].

\subsection{State of the art}

Equation (1) belong to the class of energy-transport models which have been investigated particularly in semiconductor theory [14]. The first energy-transport model for semiconductors was presented by Stratton [17]. First existence results were concerned with models with very particular diffusion coefficients (being not of the form (1)) [2,3] or with uniformly positive definite diffusion matrices [8]. Existence results for physically more realistic diffusion coefficients were shown in [6], but only for situations close to equilibrium. A degenerate energy-transport system with a simplified temperature equation was analyzed in [15]. Energy-transport models do not only appear in semiconductor theory. For instance, they have been used to model selfgravitating particle clouds [4] and the dynamics in optical lattices [5].

In [19], the global existence of weak solutions to the model

$$
\partial_{t} \rho=\Delta(\rho \theta), \quad \partial_{t}(\rho \theta)=\frac{5}{3} \Delta\left(\rho \theta^{2}\right)
$$

in a bounded domain $\Omega$ with no-flux boundary conditions was proved. At first glance, Eq. (1) look simpler than (6) because of the additional diffusion in the energy equation. However, the ideas in [19] cannot be easily applied to (1). Indeed, the key idea in [19] was to introduce the variables $u=\rho \theta$ and $v=\rho \theta^{2}$ and to apply the Stampacchia trunction method to a time-discretized version of

$$
\partial_{t}\left(\frac{u^{2}}{v}\right)=\Delta u, \quad \partial_{t} u=\frac{5}{3} \Delta v .
$$

The functionals $\int_{\Omega} \rho^{2} \theta^{b} d x$ turn out to be Lyapunov functionals along solutions to (7) for suitable values of $b \in \mathbb{R}$, leading to uniform gradient estimates. However, the additional term in the energy equation of (1) complicates the derivation of a priori estimates. Thus, the proof in [19] seems to be rather specific to system (6) and is not generalizable. Our idea is to treat (1) by combining entropy methods and tools from mathematical fluid dynamics, which may be also applied to other cross-diffusion systems.

\subsection{Mathematical key ideas}

As explained before, the first key idea is to exploit, in contrast to [19], the entropy structure of (1). Indeed, recalling the mathematical entropy density

$$
h(\rho, \theta)=\rho \log \frac{\rho}{\theta^{3 / 2}}-\log \theta \quad \text { for } \rho, \theta>0,
$$


a formal computation (which is made rigorous for an approximate scheme; see (23)) gives the entropy dissipation equation

$$
\frac{d}{d t} \int_{\Omega} h(\rho, \theta) d x+\int_{\Omega}\left(2|\nabla \sqrt{\rho \theta}|^{2}+|\nabla \log \theta|^{2}\left(1+\frac{5}{2} \rho \theta\right)\right) d x=0,
$$

which provides $H^{1}(\Omega)$ estimates for $\sqrt{\rho \theta}$ and $\log \theta$. Moreover, this estimate implies that $\theta>0$ a.e. (but not $\rho>0$ ).

Clearly, the entropy estimates are not sufficient to pass to the de-regularization limit in the approximate scheme. Further bounds are derived from the $H^{-1}(\Omega)$ method, i.e., we use basically $(-\Delta)^{-1} \rho$ and $(-\Delta)^{-1} E$, respectively, as test functions in the weak formulation of (1) (second key idea). This method gives estimates for

$$
\int_{\Omega} \rho^{2} \theta d x \quad \text { and } \quad \int_{\Omega}\left(\theta+\frac{5}{2} \rho \theta^{2}\right)\left(\theta+\frac{3}{2} \rho \theta\right) d x .
$$

Combining these bounds with those coming from the entropy inequality and the conservation laws leads to estimates for $\nabla(\rho \theta)=\sqrt{\rho \theta} \nabla \sqrt{\rho \theta}, \nabla \theta=\theta \nabla \log \theta$ and consequently for $E$ in $W^{1,1}(\Omega)$. Moreover, $\partial_{t} E$ is bounded in some dual Sobolev space. This allows us to apply the Aubin-Lions lemma to E. Unfortunately, we do not obtain gradient estimates for $\rho$.

To overcome this issue, we use tools from mathematical fluid dynamics (third key idea). Let $\left(\rho_{\delta}, \theta_{\delta}\right)$ be approximate solutions to (1) (in a sense made precise in Sect. 3). First, we write the mass balance equation in the renormalized form

$$
\partial_{t} f\left(\rho_{\delta}\right)-\operatorname{div}\left(f^{\prime}\left(\rho_{\delta}\right) \nabla\left(\rho_{\delta} \theta_{\delta}\right)\right)=-f^{\prime \prime}\left(\rho_{\delta}\right) \nabla \rho_{\delta} \cdot \nabla\left(\rho_{\delta} \theta_{\delta}\right)
$$

in the sense of distributions for smooth functions $f$ with bounded derivatives. Let $g$ another smooth function with bounded derivatives and introduce the vectors

$$
U_{\delta}=\left(f\left(\rho_{\delta}\right),-f^{\prime}\left(\rho_{\delta}\right) \nabla\left(\rho_{\delta} \theta_{\delta}\right)\right), \quad V_{\delta}=\left(g\left(\theta_{\delta}\right), 0,0,0\right) .
$$

We deduce from the properties of $f$ and $g$ and the a priori estimates that $\operatorname{div}_{(t, x)} U_{\delta}$ and $\operatorname{curl}_{(t, x)} V_{\delta}$ are uniformly bounded in $L^{1}(\Omega \times(0, T))$ and hence relatively compact in $W^{-1, r}(\Omega)$ for some $r>1$. The div-curl lemma implies that $\overline{U_{\delta} \cdot V_{\delta}}=\overline{U_{\delta}} \cdot \overline{V_{\delta}}$ a.e., where the bar denotes the weak limit of the corresponding sequence. Thus, $\overline{f\left(\rho_{\delta}\right) g\left(\theta_{\delta}\right)}=\overline{f\left(\rho_{\delta}\right)} \overline{g\left(\theta_{\delta}\right)}$ a.e. A truncation procedure yields that $\overline{\rho_{\delta} \theta_{\delta}}=\rho \theta$, where $\rho$ and $\theta$ are the weak limits of $\left(\rho_{\delta}\right)$ and $\left(\theta_{\delta}\right)$, respectively. As $\left(E_{\delta}\right)$ converges strongly, by the Aubin-Lions lemma, we are able to prove that $\theta_{\delta} \rightarrow \theta$ and eventually $\rho_{\delta} \rightarrow \rho$ a.e. These limits allow us to identify the weak limits and to pass to the limit $\delta \rightarrow 0$ in the approximate equations. The approximate scheme contains additional terms which need to be treated carefully such that our arguments are more technical than presented here. In fact, we need three approximation levels; see Sect. 3 for details. 


\subsection{Main result}

Our main result is as follows:

Theorem 1. (Existence of weak solutions). Let $\Omega \subset \mathbb{R}^{3}$ be a bounded domain with $\partial \Omega \in C^{1,1}$. Let $\rho^{0}, \theta^{0} \in L^{1}(\Omega)$ satisfy $\rho^{0} \geq 0, \theta^{0} \geq 0$ in $\Omega$ and $\rho^{0} \theta^{0}$, $h\left(\rho^{0}, \theta^{0}\right) \in L^{1}(\Omega)$, where $h$ is defined in $(8)$. Let $T>0$ and $\Omega_{T}=\Omega \times(0, T)$. Then there exist $\rho, \theta \in L^{\infty}\left(0, T ; L^{1}(\Omega)\right)$ such that

$$
\begin{aligned}
& \rho \log \rho \in L^{\infty}\left(0, T ; L^{1}(\Omega)\right), \quad E=\theta+\frac{3}{2} \rho \theta \in L^{\infty}\left(0, T ; L^{1}(\Omega)\right) \cap L^{2}\left(\Omega_{T}\right), \\
& \sqrt{\rho \theta}, \log \theta \in L^{2}\left(0, T ; H^{1}(\Omega)\right), \quad \rho \theta^{2} \in L^{3 / 2}\left(\Omega_{T}\right), \\
& \partial_{t} \rho \in L^{4 / 3}\left(0, T ; W^{1,4}(\Omega)^{\prime}\right), \quad \partial_{t} E \in L^{6 / 5}\left(0, T ; W^{2,4}(\Omega)^{\prime}\right) ;
\end{aligned}
$$

it holds that $\rho \geq 0$ and $\theta>0$ a.e. in $\Omega_{T} ;(\rho, \theta)$ is a weak solution to (1)-(3) in the sense

$$
\begin{array}{r}
\int_{0}^{T}\left\langle\partial_{t} \rho, \psi_{1}\right\rangle d t+\frac{3}{2} \int_{0}^{T} \int_{\Omega} \nabla(\rho \theta) \cdot \nabla \psi_{1} d x d t=0 \\
\int_{0}^{T}\left\langle\partial_{t} E, \psi_{2}\right\rangle d t-\int_{0}^{T} \int_{\Omega}\left(\theta+\frac{5}{2} \rho \theta^{2}\right) \Delta \psi_{2} d x d t=0
\end{array}
$$

for any test functions $\psi_{1} \in L^{4}\left(0, T ; W^{1,4}(\Omega)\right), \psi_{2} \in L^{6}\left(0, T ; W^{2,4}(\Omega)\right)$; and the initial data (3) is satisfied in the sense of $W^{1,4}(\Omega)^{\prime}$ and $W^{2,4}(\Omega)^{\prime}$, respectively. Moreover, the total mass and energy are preserved:

$$
\int_{\Omega} \rho(t) d x=\int_{\Omega} \rho^{0} d x, \quad \int_{\Omega} E(t) d x=\int_{\Omega} E^{0} d x \quad \text { for } t \geq 0 .
$$

In the theorem, we denote by $X^{\prime}$ the dual space of the Banach space $X$.

The paper is organized as follows. Equation (1) are formally derived from a relaxation-time kinetic model in Sect. 2, while the proof of Theorem 1 is presented in Sect. 3.

\section{Formal derivation from a kinetic model}

We consider a gas which is rarefied enough such that collisions between gas particles can be neglected, but there are thermalizing collisions at a fixed rate with a nonmoving background. This is modeled by sampling post-collisional velocities from a Maxwellian distribution with zero mean velocity and with the background temperature, which is determined from the assumptions of energy conservation as well as heat transport in the background governed by the Fourier law. These assumptions lead to the equations

$$
\begin{aligned}
\varepsilon^{2} \partial_{t} f_{\varepsilon}+\varepsilon v \cdot \nabla f_{\varepsilon} & =\rho_{\varepsilon} M\left(\theta_{\varepsilon}\right)-f_{\varepsilon}, \\
\varepsilon^{2}\left(\partial_{t} \theta_{\varepsilon}-\Delta \theta_{\varepsilon}\right) & =\frac{1}{2} \int_{\mathbb{R}^{3}}|v|^{2}\left(f_{\varepsilon}-\rho_{\varepsilon} M\left(\theta_{\varepsilon}\right)\right) d v,
\end{aligned}
$$

which are written in dimensionless form with a diffusive macroscopic scaling with the scaled Knudsen number $0<\varepsilon \ll 1$. The gas is described by the distribution function $f_{\varepsilon}(x, v, t)$ with the velocity $v \in \mathbb{R}^{3}$, and the temperature 
of the background is $\theta_{\varepsilon}(x, t)$. The gradient and Laplace operators are meant with respect to the position variable $x$, and the Maxwellian is given by

$$
M(\theta ; v)=\frac{1}{(2 \pi \theta)^{3 / 2}} \exp \left(-\frac{|v|^{2}}{2 \theta}\right) .
$$

Finally, the position density of the gas is defined by

$$
\rho_{\varepsilon}(x, t)=\int_{\mathbb{R}^{3}} f_{\varepsilon}(x, v, t) d v .
$$

The right-hand side of the heat equation (12) has been chosen such that the sum of the kinetic energy of the gas and the thermal energy of the background is conserved. In [12], the energy-transport system (1) has been derived formally from $(11)-(12)$ in the macroscopic limit $\varepsilon \rightarrow 0$. We repeat the argument here for completeness.

In the computations, the moments of the Maxwellian up to order 4 will be needed:

$$
\begin{aligned}
& \int_{\mathbb{R}^{3}} M(\theta ; v) d v=1, \quad \int_{\mathbb{R}^{3}} v M(\theta ; v) d v=\int_{\mathbb{R}^{3}} v|v|^{2} M(\theta ; v) d v=0, \\
& \int_{\mathbb{R}^{3}} v_{i} v_{j} M(\theta ; v) d v=\theta \delta_{i j}, \quad \int_{\mathbb{R}^{3}} v_{i} v_{j}|v|^{2} M(\theta ; v) d v=5 \theta^{2} \delta_{i j},
\end{aligned}
$$

where $v_{i}, v_{j}$ denote the components of $v(i, j=1,2,3)$. From (11)-(12), the local conservation laws for mass and energy,

$$
\begin{aligned}
& \partial_{t} \rho_{\varepsilon}+\operatorname{div}\left(\frac{1}{\varepsilon} \int_{\mathbb{R}^{3}} v f_{\varepsilon} d v\right)=0, \\
& \partial_{t}\left(\theta_{\varepsilon}+\frac{1}{2} \int_{\mathbb{R}^{3}}|v|^{2} f_{\varepsilon} d v\right)+\operatorname{div}\left(\frac{1}{2 \varepsilon} \int_{\mathbb{R}^{3}} v|v|^{2} f_{\varepsilon} d v-\nabla \theta_{\varepsilon}\right)=0,
\end{aligned}
$$

can be derived by integration of (11) with respect to $v$ and, respectively, by integration of (11) against $|v|^{2} / 2$ and adding to (12).

In a formal convergence analysis, we assume $f_{\varepsilon} \rightarrow f, \rho_{\varepsilon} \rightarrow \rho$, and $\theta_{\varepsilon} \rightarrow \theta$ as $\varepsilon \rightarrow 0$ and deduce from (11) that $f=\rho M(\theta)$. With (14), we obtain for the kinetic energy density

$$
\lim _{\varepsilon \rightarrow 0} \frac{1}{2} \int_{\mathbb{R}^{3}}|v|^{2} f_{\varepsilon} d v=\frac{3}{2} \rho \theta .
$$

The limit of the mass flux is obtained by multiplication of (11) by $v / \varepsilon$, integration with respect to $v$, and passing to the limit, using again (14):

$$
\begin{aligned}
\lim _{\varepsilon \rightarrow 0}\left(\frac{1}{\varepsilon} \int_{\mathbb{R}^{3}} v f_{\varepsilon} d v\right) & =-\int_{\mathbb{R}^{3}} v(v \cdot \nabla(\rho M(\theta ; v))) d v \\
& =-\operatorname{div}\left(\rho \int_{\mathbb{R}^{3}} v \otimes v M(\theta ; v) d v\right)=-\nabla(\rho \theta) .
\end{aligned}
$$


Analogously, we compute the flux of the kinetic energy,

$$
\begin{aligned}
\lim _{\varepsilon \rightarrow 0}\left(\frac{1}{2 \varepsilon} \int_{\mathbb{R}^{3}} v_{i}|v|^{2} f_{\varepsilon} d v\right) & =-\frac{1}{2} \int_{\mathbb{R}^{3}} v_{i}|v|^{2}(v \cdot \nabla(\rho M(\theta ; v))) d v \\
& =-\frac{1}{2} \sum_{j=1}^{3} \frac{\partial}{\partial x_{j}}\left(\rho \int_{\mathbb{R}^{3}} v_{i} v_{j}|v|^{2} M(\theta ; v) d v\right)=-\frac{5}{2} \frac{\partial}{\partial x_{i}}\left(\rho \theta^{2}\right)
\end{aligned}
$$

for $i=1,2,3$. Using these results in the limits of the conservation laws leads to $(1)$.

\section{Proof of Theorem 1}

We approximate Eq. (1) in the following way. The time derivative is replaced by the implicit Euler discretization with parameter $\tau>0$. This is needed to avoid issues related to the time regularity. A higher-order $H^{4}$ regularization for $\phi=\partial h / \partial \rho$ in the mass balance equation with parameter $\varepsilon>0$ gives $H^{2}(\Omega)$ regularity and compactness in $W^{1,4}(\Omega)$. Furthermore, $H^{2}(\Omega)$ and $W^{1,4}(\Omega)$ regularizations for $\log \theta$ with the same parameter are added to the energy balance equation. The $W^{1,4}(\Omega)$ regularization is needed to derive estimates when using both $\log \theta$ and $-1 / \theta$ as test functions in (1). Furthermore, we add an additional $H^{2}(\Omega)$ regularization for $\phi$ in the mass balance equation with parameter $\delta>0$, which removes the degeneracy of the diffusion matrix $M$ in (4). Finally, we add the artificial heat flux $\Delta \theta^{3}$ in the energy density equation with the same parameter $\delta$ to obtain gradient estimates for the temperature, and we add the term $\theta^{-N} \log \theta$ for some $N>0$ to achieve an estimate for $\theta^{-(N+1)}$.

After having proved the existence of solutions to the approximate problem and some a priori estimates coming from the entropy inequality, we perform the limits $\varepsilon \rightarrow 0, \tau \rightarrow 0$, and $\delta \rightarrow 0$ (in this order).

\subsection{Solution of the approximate problem}

We wish to solve a system which approximates (1) and is formulated in the variables $\phi$ and $w=\log \theta$, similarly as in (4). We interpret $\rho$ and $E=\theta\left(1+\frac{3}{2} \rho\right)$ as functions of $(\phi, w)$, i.e.

$$
\rho(\phi, w)=\exp \left(\phi+\frac{3}{2} w-\frac{5}{2}\right), \quad E(\rho, w)=\left(1+\frac{3}{2} \rho(\phi, w)\right) \exp (w) .
$$

In this notation, the diffusion coefficients become

$$
M_{11}=\rho e^{w}, \quad M_{12}=\frac{5}{2} \rho e^{2 w}, \quad M_{22}=e^{2 w}\left(1+\frac{35}{4} \rho e^{w}\right) .
$$

Let $T>0$ and let the approximation parameters $\tau>0$ (such that $T / \tau \in$ $\mathbb{N}), \varepsilon>0$, and $\delta>0$ be given. Furthermore, let $0<N<5$ be a number needed for the approximation $\theta^{-N} \log \theta$ in the energy balance equation. 
We wish to find $\left(\phi^{k}, w^{k}\right) \in H^{2}\left(\Omega ; \mathbb{R}^{2}\right)$ such that, with $\rho^{k}=\rho\left(\phi^{k}, w^{k}\right)$, $E^{k}=E\left(\rho^{k}, w^{k}\right)$,

$$
\begin{aligned}
0= & \frac{1}{\tau} \int_{\Omega}\left(\rho^{k}-\rho^{k-1}\right) \psi_{1} d x+\int_{\Omega}\left(M_{11}^{k} \nabla \phi^{k}+M_{12}^{k} e^{-w^{k}} \nabla w^{k}\right) \cdot \nabla \psi_{1} d x \\
& +\varepsilon \int_{\Omega} D^{2} \phi^{k}: D^{2} \psi_{1} d x+\delta \int_{\Omega}\left(\nabla \phi^{k} \cdot \nabla \psi_{1}+\phi^{k} \psi_{1}\right) d x \\
0= & \frac{1}{\tau} \int_{\Omega}\left(E^{k}-E^{k-1}\right) \psi_{2} d x+\int_{\Omega}\left(M_{12}^{k} \nabla \phi^{k}+M_{22}^{k} e^{-w^{k}} \nabla w^{k}\right) \cdot \nabla \psi_{2} d x \\
& +\varepsilon \int_{\Omega} e^{w^{k}}\left(D^{2} w^{k}: D^{2} \psi_{2}+\left|\nabla w^{k}\right|^{2} \nabla w^{k} \cdot \nabla \psi_{2}\right) d x+\varepsilon \int_{\Omega}\left(1+e^{w^{k}}\right) w^{k} \psi_{2} d x \\
& +\delta \int_{\Omega} e^{3 w^{k}} \nabla w^{k} \cdot \nabla \psi_{2} d x+\delta \int_{\Omega} e^{-N w^{k}} w^{k} \psi_{2} d x
\end{aligned}
$$

for all $\left(\psi_{1}, \psi_{2}\right) \in H^{2}\left(\Omega ; \mathbb{R}^{2}\right)$, and $M_{i j}^{k}$ are given by (15) with $(\rho, w)$ replaced by $\left(\rho^{k}, w^{k}\right)$. The existence of solutions to (16)-(17) is shown in two steps.

Step 1: solution of the linearized approximated problem. In the following, we drop the superindex $k$. Let $(\widetilde{\phi}, \widetilde{w}) \in W^{1,4}\left(\Omega ; \mathbb{R}^{2}\right)$ be given and set $\widetilde{\rho}=$ $\rho(\widetilde{\phi}, \widetilde{w}), \widetilde{E}=E(\widetilde{\phi}, \widetilde{w})$. We wish to find $(\phi, w) \in H^{2}\left(\Omega ; \mathbb{R}^{2}\right)$ such that

$$
a_{1}\left(\phi, \psi_{1}\right)=\sigma F_{1}\left(\psi_{1}\right), \quad a_{2}\left(w, \psi_{2}\right)=\sigma F_{2}\left(\psi_{2}\right)
$$

for all $\left(\psi_{1}, \psi_{2}\right) \in H^{2}\left(\Omega ; \mathbb{R}^{2}\right)$, where $\sigma \in[0,1]$ and

$$
\begin{aligned}
a_{1}\left(\phi, \psi_{1}\right)= & \varepsilon \int_{\Omega} D^{2} \phi: D^{2} \psi_{1} d x+\delta \int_{\Omega}\left(\nabla \phi \cdot \nabla \psi_{1}+\phi \psi_{1}\right) d x, \\
a_{2}\left(w, \psi_{2}\right)= & \varepsilon \int_{\Omega} e^{\widetilde{w}}\left(D^{2} w: D^{2} \psi_{2}+|\nabla \widetilde{w}|^{2} \nabla w \cdot \nabla \psi_{2}\right) d x+\varepsilon \int_{\Omega}\left(1+e^{\widetilde{w}}\right) w \psi_{2} d x \\
& +\delta \int_{\Omega} e^{3 \widetilde{w}} \nabla w \cdot \nabla \psi_{2} d x+\delta \int_{\Omega} e^{-N \widetilde{w}} w \psi_{2} d x, \\
F_{1}\left(\psi_{1}\right)= & -\frac{1}{\tau} \int_{\Omega}\left(\widetilde{\rho}-\rho^{k-1}\right) \psi_{1} d x-\int_{\Omega}\left(\widetilde{M}_{11} \nabla \widetilde{\phi}+\widetilde{M}_{12} e^{-\widetilde{w}} \nabla \widetilde{w}\right) \cdot \nabla \psi_{1} d x \\
F_{2}\left(\psi_{2}\right)= & -\frac{1}{\tau} \int_{\Omega}\left(\widetilde{E}-E^{k-1}\right) \psi_{2} d x-\int_{\Omega}\left(\widetilde{M}_{12} \nabla \widetilde{\phi}+\widetilde{M}_{22} e^{-\widetilde{w}} \nabla \widetilde{w}\right) \cdot \nabla \psi_{2} d x,
\end{aligned}
$$

where $\widetilde{M}_{i j}$ is given by $(15)$ with $(\rho, w)$ replaced by $(\widetilde{\rho}, \widetilde{w})$. The bilinear forms $a_{1}$ and $a_{2}$ are coercive on $H^{2}(\Omega)$ since, by the generalized Poincaré inequality [18, Chap. 2, Sect. 1.4],

$$
\begin{aligned}
& a_{1}(\phi, \phi)=\varepsilon \int_{\Omega}\left|D^{2} \phi\right|^{2} d x+\delta \int_{\Omega}\left(|\nabla \phi|^{2}+\phi^{2}\right) d x \geq \min \{\varepsilon, \delta\}\|\phi\|_{H^{2}(\Omega)}^{2}, \\
& a_{2}(w, w) \geq \varepsilon \int_{\Omega}\left(C\left|D^{2} w\right|^{2}+w^{2}\right) d x \geq \varepsilon C\|w\|_{H^{2}(\Omega)}^{2}
\end{aligned}
$$

for some constant $C>0$. The linear forms $F_{1}$ and $F_{2}$ are continuous on $H^{2}(\Omega)$ since, by the continuous embedding $W^{1,4}(\Omega) \hookrightarrow L^{\infty}(\Omega), \widetilde{\phi}$ and $\widetilde{w}$ are $L^{\infty}(\Omega)$ functions such that $\widetilde{\rho}, \widetilde{E} \in L^{\infty}(\Omega)$ too. The Lax-Milgram lemma implies the existence of a unique solution $(\phi, w)$ to $(18)$ such that $\rho=\rho(\phi, w)>0$ and 
$E=E(\phi, w)>0$. This defines the fixed-point operator $S: W^{1,4}\left(\Omega ; \mathbb{R}^{2}\right) \times$ $[0,1] \rightarrow W^{1,4}\left(\Omega ; \mathbb{R}^{2}\right), S(\widetilde{\phi}, \widetilde{w}, \sigma)=(\phi, w)$, where $(\phi, w)$ solves $(18)$.

Step 2: solution of the approximate problem. We wish to apply the LeraySchauder fixed-point theorem. It holds that $S(\widetilde{\phi}, \widetilde{w}, 0)=0$. Standard arguments show that $S: W^{1,4}\left(\Omega ; \mathbb{R}^{2}\right) \rightarrow H^{2}\left(\Omega ; \mathbb{R}^{2}\right)$ is continuous. Since $H^{2}\left(\Omega ; \mathbb{R}^{2}\right)$ is compactly embedded into $W^{1,4}\left(\Omega ; \mathbb{R}^{2}\right), S: W^{1,4}\left(\Omega ; \mathbb{R}^{2}\right) \rightarrow W^{1,4}\left(\Omega ; \mathbb{R}^{2}\right)$ is compact. It remains to show that there exists a uniform bound in $W^{1,4}\left(\Omega ; \mathbb{R}^{2}\right)$ for all fixed points.

Let $\sigma \in(0,1]$ and let $(\phi, w)$ be a fixed point of $S(\cdot, \cdot, \sigma)$. It is a solution to (16)-(17) with $\phi=\phi^{k}, w=w^{k}, \rho=\rho^{k}$, and $E=E^{k}$. We use the test functions $\psi_{1}=\phi$ and $\psi_{2}=1-e^{-w}$ in (16) and (17), respectively, and add both equations. (We use $1-e^{-w}$ instead of $-e^{-w}$ as a test function in order to be able to treat the term $\varepsilon \int_{\Omega}\left(1+e^{w}\right) w \psi_{2} d x$ and to obtain the entropy and energy balance in one single equation.) Then

$$
\begin{aligned}
0= & \frac{\sigma}{\tau} \int_{\Omega}\left(\left(\rho-\rho^{k-1}\right) \phi+\left(E-E^{k-1}\right)\left(1-e^{-w}\right)\right) d x \\
& +\int_{\Omega}\left(M_{11}|\nabla \phi|^{2}+2 M_{12} e^{-w} \nabla \phi \cdot \nabla w+M_{22} e^{-2 w}|\nabla w|^{2}\right) d x \\
& +\varepsilon \int_{\Omega} e^{w}\left(D^{2} w: D^{2}\left(-e^{-w}\right)+e^{-w}|\nabla w|^{4}\right) d x+\delta \int_{\Omega} e^{2 w}|\nabla w|^{2} d x \\
& +\varepsilon \int_{\Omega}\left|D^{2} \phi\right|^{2} d x+\delta \int_{\Omega}\left(|\nabla \phi|^{2}+\phi^{2}\right) d x+\varepsilon \int_{\Omega}\left(1+e^{w}\right) w\left(1-e^{-w}\right) d x \\
& +\delta \int_{\Omega} e^{-(N+1) w} w\left(e^{w}-1\right) d x \\
= & : I_{1}+\cdots+I_{8} .
\end{aligned}
$$

To estimate the first integral $I_{1}$, we use the entropy density (8), formulated in terms of the variables $(\rho, E)$,

$$
h(\rho, \theta)=\widetilde{h}(\rho, E)=\rho \log \rho+\left(1+\frac{3}{2} \rho\right) \log \frac{E}{1+\frac{3}{2} \rho} .
$$

The function $\widetilde{h}$ in the variables $(\rho, E)$ is convex, since the determinant of its Hessian,

$$
D^{2} \widetilde{h}(\rho, E)=\left(\begin{array}{cc}
\frac{1}{\rho}+\frac{9}{4}\left(1+\frac{3}{2} \rho\right)^{-1} & -\frac{3}{2} \frac{1}{E} \\
-\frac{3}{2} \frac{1}{E} & \left(1+\frac{3}{2} \rho\right) E^{-2}
\end{array}\right)
$$

equals $\left(1+\frac{3}{2} \rho\right) /\left(\rho E^{2}\right)$, which is positive. This implies that

$$
\widetilde{h}\left(\rho_{1}, E_{1}\right)-\widetilde{h}\left(\rho_{2}, E_{2}\right) \leq D \widetilde{h}\left(\rho_{1}, E_{1}\right) \cdot\left(\begin{array}{c}
\rho_{1}-\rho_{2} \\
E_{1}-E_{2}
\end{array}\right)=\left(\rho_{1}-\rho_{2}\right) \phi+\left(E_{1}-E_{2}\right)\left(-e^{-w}\right)
$$

for any $\left(\rho_{1}, E_{2}\right),\left(\rho_{2}, E_{2}\right)>0$, and consequently,

$$
I_{1} \geq \frac{\sigma}{\tau} \int_{\Omega}\left(\widetilde{h}(\rho, E)-\widetilde{h}\left(\rho^{k-1}, E^{k-1}\right)\right) d x+\frac{\sigma}{\tau} \int_{\Omega}\left(E-E^{k-1}\right) d x .
$$


The second integral $I_{2}$ is nonnegative since

$$
\begin{aligned}
& M_{11}|\nabla \phi|^{2}+2 M_{12} e^{-w} \nabla \phi \cdot \nabla w+M_{22} e^{-2 w}|\nabla w|^{2} \\
& =\rho e^{w}|\nabla \phi|^{2}+5 \rho e^{w} \nabla \phi \cdot \nabla w+\left(1+\frac{35}{4} \rho e^{w}\right)|\nabla w|^{2} \\
& \quad=\rho e^{w}\left(\frac{1}{8}|\nabla \phi|^{2}+\frac{7}{8}\left|\nabla \phi+\frac{20}{7} \nabla w\right|^{2}+\frac{45}{28}|\nabla w|^{2}\right)+|\nabla w|^{2} \\
& \quad \geq \frac{1}{8} \rho e^{w}|\nabla \phi|^{2}+\left(1+\rho e^{w}\right)|\nabla w|^{2} .
\end{aligned}
$$

The integrals $I_{3}, I_{7}$, and $I_{8}$ are estimated according to

$$
\begin{aligned}
I_{3} & =\frac{1}{2}\left(\left|D^{2} w\right|^{2}+\left|D^{2} w-\nabla w \otimes \nabla w\right|^{2}+|\nabla w|^{4}\right) \geq \frac{1}{2}\left(\left|D^{2} w\right|^{2}+|\nabla w|^{4}\right), \\
I_{7} & =2 \varepsilon \int_{\Omega} w \sinh (w) d x \geq \varepsilon \int_{\Omega} w^{2} d x \\
I_{8} & =\delta \int_{\Omega} e^{-(N+1) w} w\left(e^{w}-1\right) d x \geq \delta \int_{\Omega} e^{-(N+1) w} 1_{\{w>-2\}} d x \\
& =\delta \int_{\Omega} e^{-(N+1) w} d x-\delta \int_{\{w \geq-2\}} e^{-(N+1) w} d x \\
& \geq \delta \int_{\Omega} e^{-(N+1) w} d x-\delta e^{2(N+1)} \operatorname{meas}(\Omega) .
\end{aligned}
$$

Therefore, we obtain from (19)

$$
\begin{aligned}
& \frac{\sigma}{\tau} \int_{\Omega}(\widetilde{h}(\rho, E)+E) d x+\sigma \int_{\Omega}\left\{\frac{1}{8} \rho e^{w}|\nabla \phi|^{2}+\left(1+\rho e^{w}\right)|\nabla w|^{2}\right\} d x \\
& \quad+\varepsilon \int_{\Omega}\left|D^{2} \phi\right|^{2} d x+\frac{\varepsilon}{2} \int_{\Omega}\left(\left|D^{2} w\right|^{2}+|\nabla w|^{4}+w^{2}\right) d x \\
& \quad+\delta \int_{\Omega} e^{2 w}|\nabla w|^{2} d x+\delta \int_{\Omega}\left(|\nabla \phi|^{2}+\phi^{2}\right) d x+\delta \int_{\Omega} e^{-(N+1) w} d x \\
& \leq \frac{\sigma}{\tau} \int_{\Omega}\left(\widetilde{h}\left(\rho^{k-1}, E^{k-1}\right)+E^{k-1}\right) d x+C \delta
\end{aligned}
$$

where $C>0$ is here and in the following a generic constant independent of $\tau, \varepsilon$, and $\delta$. This gives a uniform $H^{2}(\Omega)$ estimate for $\phi$ and $w$, independent of $\sigma$ (but depending on $\varepsilon$ and $\delta$ ), and hence the desired uniform estimate for $(\phi, w)$ in $W^{1,4}\left(\Omega ; \mathbb{R}^{2}\right)$. By the Leray-Schauder fixed-point theorem, there exists a solution $\left(\phi^{k}, w^{k}\right):=(\phi, w) \in H^{2}\left(\Omega ; \mathbb{R}^{2}\right)$ to $(16)-(17)$ with $\sigma=1$, $\rho^{k}=\rho\left(\phi^{k}, w^{k}\right)$, and $E^{k}=E\left(\phi^{k}, w^{k}\right)$. Moreover, this solution satisfies (20) with $\sigma=1$. 
We reformulate Eqs. (16)-(17) by inserting definition (15) of the diffusion coefficients and computing (we drop the superindex $k$ )

$$
\begin{aligned}
M_{11} \nabla \phi+M_{12} e^{-w} \nabla w & =\rho \theta \nabla\left(\log \rho-\frac{3}{2} \log \theta\right)+\frac{5}{2} \rho \nabla \theta=\nabla(\rho \theta), \\
M_{12} \nabla \phi+M_{22} e^{-w} \nabla w & =\frac{5}{2} \rho \theta^{2} \nabla\left(\log \rho-\frac{3}{2} \log \theta\right)+\left(1+\frac{35}{4} \rho \theta\right) \nabla \theta \\
& =\nabla\left(\theta+\frac{5}{2} \rho \theta^{2}\right) .
\end{aligned}
$$

Therefore, $\left(\phi^{k}, \rho^{k}, \theta^{k}, w^{k}\right)$ solves

$$
\begin{aligned}
0= & \frac{1}{\tau} \int_{\Omega}\left(\rho^{k}-\rho^{k-1}\right) \psi_{1} d x+\int_{\Omega} \nabla\left(\rho^{k} \theta^{k}\right) \cdot \nabla \psi_{1} d x+\varepsilon \int_{\Omega} D^{2} \phi^{k}: D^{2} \psi_{1} d x \\
& +\delta \int_{\Omega}\left(\nabla \phi^{k} \cdot \nabla \psi_{1}+\phi^{k} \psi_{1}\right) d x \\
0= & \frac{1}{\tau} \int_{\Omega}\left(E^{k}-E^{k-1}\right) \psi_{2} d x+\int_{\Omega} \nabla\left(\theta^{k}+\frac{5}{2} \rho^{k}\left(\theta^{k}\right)^{2}\right) \cdot \nabla \psi_{2} d x \\
& +\delta \int_{\Omega} e^{3 w^{k}} \nabla w^{k} \cdot \nabla \psi_{2} d x+\varepsilon \int_{\Omega}\left(1+e^{w^{k}}\right) w^{k} \psi_{2} d x \\
& +\varepsilon \int_{\Omega} e^{w^{k}}\left(D^{2} w^{k}: D^{2} \psi_{2}+\left|\nabla w^{k}\right|^{2} \nabla w^{k} \cdot \nabla \psi_{2}\right) d x+\delta \int_{\Omega} e^{-N w^{k}} w^{k} \psi_{2} d x
\end{aligned}
$$

for test functions $\psi_{1}, \psi_{2} \in H^{2}(\Omega)$.

\subsection{Uniform estimates}

Set $\theta^{k-1}=\exp \left(w^{k-1}\right)$ and $\theta^{k}=\exp \left(w^{k}\right)$. In the following, we drop again the superindex $k$ to simplify the notation. We reformulate inequality (20) to obtain gradient estimates for expressions depending on $\rho$ and $\theta$. We estimate the second integral in (20):

$$
\begin{aligned}
\frac{1}{8} \rho e^{w}|\nabla \phi|^{2}+\left(1+\rho e^{w}\right)|\nabla w|^{2} \\
=|\nabla \log \theta|^{2}+\frac{1}{8} \rho \theta\left|\frac{\nabla \rho}{\rho}-\frac{3}{2} \frac{\nabla \theta}{\theta}\right|^{2}+\rho \frac{|\nabla \theta|^{2}}{\theta} \\
=|\nabla \log \theta|^{2}+\frac{1}{8} \rho \theta\left(\frac{|\nabla \rho|^{2}}{\rho^{2}}-3 \frac{\nabla \rho}{\rho} \cdot \frac{\nabla \theta}{\theta}+\frac{41}{4} \frac{|\nabla \theta|^{2}}{\theta^{2}}\right) \\
\geq|\nabla \log \theta|^{2}+\frac{1}{16} \rho \theta\left(\frac{|\nabla \rho|^{2}}{\rho^{2}}+\frac{|\nabla \theta|^{2}}{\theta^{2}}\right) \\
=|\nabla \log \theta|^{2}+\frac{1}{32} \rho \theta\left(\frac{|\nabla \rho|^{2}}{\rho^{2}}+\frac{|\nabla \theta|^{2}}{\theta^{2}}\right) \\
\quad+\frac{1}{32}|\sqrt{\theta} \nabla \sqrt{\rho}+\sqrt{\rho} \nabla \sqrt{\theta}|^{2}+\frac{1}{32}|\sqrt{\theta} \nabla \sqrt{\rho}-\sqrt{\rho} \nabla \sqrt{\theta}|^{2} \\
\geq|\nabla \log \theta|^{2}+\frac{1}{8} \theta|\nabla \sqrt{\rho}|^{2}+\frac{1}{32}|\nabla \sqrt{\rho \theta}|^{2} .
\end{aligned}
$$


We infer from (20) with $\sigma=1$ the reformulated discrete entropy inequality

$$
\begin{aligned}
\frac{1}{\tau} \int_{\Omega}( & \widetilde{h}(\rho, E)+E) d x+\int_{\Omega}|\nabla \log \theta|^{2} d x+\frac{1}{8} \int_{\Omega} \theta|\nabla \sqrt{\rho}|^{2} d x+\frac{1}{64} \int_{\Omega}|\nabla \sqrt{\rho \theta}|^{2} d x \\
& +\varepsilon \int_{\Omega}\left|D^{2} \phi\right|^{2} d x+\frac{\varepsilon}{2} \int_{\Omega}\left(\left|D^{2} w\right|^{2}+|\nabla w|^{4}+w^{2}\right) d x \\
& +\delta \int_{\Omega}\left(|\nabla \phi|^{2}+\phi^{2}\right) d x+\delta \int_{\Omega}\left|\nabla e^{w}\right|^{2} d x+\delta \int_{\Omega} e^{-(N+1) w} d x \\
\leq & \frac{1}{\tau} \int_{\Omega}\left(\widetilde{h}\left(\rho^{k-1}, E^{k-1}\right)+E^{k-1}\right) d x+\delta C .
\end{aligned}
$$

There exists $c \in(0,1)$ such that $x-\log x \geq c(x+|\log x|)$ for all $x>0$. Therefore,

$$
\begin{aligned}
\widetilde{h}(\rho, E)+E & =\rho \log \rho-\frac{3}{2} \rho \log \theta-\log \theta+\left(1+\frac{3}{2} \rho\right) \theta \\
& =\rho \log \rho+\left(1+\frac{3}{2} \rho\right)(\theta-\log \theta) \geq \rho \log \rho+c(1+\rho)(\theta+|\log \theta|) .
\end{aligned}
$$

This provides the following uniform estimates independent of $(\delta, \varepsilon, \tau)$ :

$$
\|\rho \log \rho\|_{L^{1}(\Omega)}+\|\theta\|_{L^{1}(\Omega)}+\|\rho \theta\|_{L^{1}(\Omega)}+\|\log \theta\|_{L^{1}(\Omega)} \leq C .
$$

\subsection{Limit $\varepsilon \rightarrow 0$}

Let $\phi_{\varepsilon}=\phi^{k}, w_{\varepsilon}=w^{k}$ be a solution to (16)-(17). We set $\rho_{\varepsilon}=\rho\left(\phi_{\varepsilon}, w_{\varepsilon}\right)$, $E_{\varepsilon}=E\left(\rho_{\varepsilon}, w_{\varepsilon}\right), \theta_{\varepsilon}=\exp \left(w_{\varepsilon}\right)$, and $\phi_{\varepsilon}=\log \left(\rho_{\varepsilon} / \theta_{\varepsilon}^{3 / 2}\right)+5 / 2$. We deduce from (23) and (24) the following bounds which are independent of $\varepsilon$ and $\delta$ (but not of $\tau)$ :

$$
\begin{aligned}
&\left\|\rho_{\varepsilon} \log \rho_{\varepsilon}\right\|_{L^{1}(\Omega)}+\left\|\theta_{\varepsilon}\right\|_{L^{1}(\Omega)}+\left\|\rho_{\varepsilon} \theta_{\varepsilon}\right\|_{L^{1}(\Omega)}+\left\|\log \theta_{\varepsilon}\right\|_{L^{1}(\Omega)} \leq C, \\
&\left\|\sqrt{\theta_{\varepsilon}} \nabla \sqrt{\rho_{\varepsilon}}\right\|_{L^{2}(\Omega)}+\left\|\nabla \sqrt{\rho_{\varepsilon} \theta_{\varepsilon}}\right\|_{L^{2}(\Omega)}+\left\|\nabla \log \theta_{\varepsilon}\right\|_{L^{2}(\Omega)} \leq C(\tau), \\
& \sqrt{\varepsilon}\left\|\phi_{\varepsilon}\right\|_{H^{2}(\Omega)}+\sqrt{\varepsilon}\left\|w_{\varepsilon}\right\|_{H^{2}(\Omega)} \leq C(\tau), \\
& \sqrt{\delta}\left\|\phi_{\varepsilon}\right\|_{H^{1}(\Omega)}+\sqrt{\delta}\left\|\nabla \theta_{\varepsilon}\right\|_{L^{2}(\Omega)}+\delta\left\|\theta_{\varepsilon}^{-(N+1)}\right\|_{L^{1}(\Omega)} \leq C(\tau) .
\end{aligned}
$$

These bounds allow us to derive further estimates. By the Poincaré inequality, we have

$$
\begin{aligned}
\left\|\theta_{\varepsilon}\right\|_{L^{2}(\Omega)} & \leq C\left\|\nabla \theta_{\varepsilon}\right\|_{L^{2}(\Omega)}+\left\|\theta_{\varepsilon}\right\|_{L^{1}(\Omega)} \leq C(\tau) \delta^{-1 / 2}, \\
\left\|\log \theta_{\varepsilon}\right\|_{L^{2}(\Omega)} & \leq C\left\|\nabla \log \theta_{\varepsilon}\right\|_{L^{2}(\Omega)}+\left\|\log \theta_{\varepsilon}\right\|_{L^{1}(\Omega)} \leq C(\tau) .
\end{aligned}
$$

This gives $\varepsilon$-uniform bounds for $\theta_{\varepsilon}$ and $\log \theta_{\varepsilon}$ in $H^{1}(\Omega)$ :

$$
\left\|\theta_{\varepsilon}\right\|_{H^{1}(\Omega)} \leq C(\tau) \delta^{-1 / 2}, \quad\left\|\log \theta_{\varepsilon}\right\|_{H^{1}(\Omega)} \leq C(\tau) .
$$

The $L^{1}(\Omega)$ bound for $\rho_{\varepsilon} \theta_{\varepsilon}$ and the $L^{2}(\Omega)$ bound for $\nabla \sqrt{\rho_{\varepsilon} \theta_{\varepsilon}}$ imply that

$$
\left\|\sqrt{\rho_{\varepsilon} \theta_{\varepsilon}}\right\|_{H^{1}(\Omega)} \leq C(\tau) .
$$

These estimates provide a uniform bound for the energy. Indeed, we deduce from the Sobolev embedding $H^{1}(\Omega) \hookrightarrow L^{6}(\Omega)$ that $\nabla\left(\rho_{\varepsilon} \theta_{\varepsilon}\right)=2 \sqrt{\rho_{\varepsilon} \theta_{\varepsilon}} \nabla \sqrt{\rho_{\varepsilon} \theta_{\varepsilon}}$ is uniformly bounded in $L^{3 / 2}(\Omega)$. This shows that $\left(E_{\varepsilon}\right)$ is bounded in $W^{1,3 / 2}(\Omega)$. 
We know that $\left(\log \theta_{\varepsilon}\right)$ and $\left(\phi_{\varepsilon}\right)$ are bounded in $H^{1}(\Omega)$. Consequently, $\log \rho_{\varepsilon}=\phi_{\varepsilon}+\frac{3}{2} \log \theta_{\varepsilon}-\frac{5}{2}$ is bounded in $H^{1}(\Omega)$ too, i.e.

$$
\sqrt{\delta}\left\|\log \rho_{\varepsilon}\right\|_{H^{1}(\Omega)} \leq C .
$$

The previous uniform bounds are sufficient to perform the limit $\varepsilon \rightarrow 0$. There exist subsequences which are not relabeled such that, as $\varepsilon \rightarrow 0$,

$\phi_{\varepsilon} \rightarrow \phi \quad$ strongly in $L^{p}(\Omega)$ and weakly in $H^{1}(\Omega)$,

$\log \rho_{\varepsilon} \rightarrow Y$ strongly in $L^{p}(\Omega)$ and weakly in $H^{1}(\Omega)$,

$\theta_{\varepsilon} \rightarrow \theta$ strongly in $L^{p}(\Omega)$ and weakly in $H^{1}(\Omega)$,

$\log \theta_{\varepsilon} \rightarrow Z$ strongly in $L^{p}(\Omega)$ and weakly in $H^{1}(\Omega)$,

$\varepsilon \phi_{\varepsilon}, \varepsilon w_{\varepsilon} \rightarrow 0$ strongly in $H^{2}(\Omega)$,

$\varepsilon^{1 / 3} \nabla w_{\varepsilon} \rightarrow 0 \quad$ strongly in $L^{4}(\Omega)$,

where $1<p<6$ and $Y, Z$ are functions in $H^{1}(\Omega)$. Up to a subsequence, we have $\log \rho_{\varepsilon} \rightarrow Y$ and $\log \theta_{\varepsilon} \rightarrow Z$ a.e. in $\Omega$. Thus, $\rho_{\varepsilon} \rightarrow e^{Y}=: \rho$ and $\theta_{\varepsilon} \rightarrow e^{Z}=: \theta$ a.e. in $\Omega$. In particular, $\rho>0$ and $\theta>0$ a.e. in $\Omega$. It follows from

$$
\int_{\left\{\rho_{\varepsilon} \geq R\right\}} \rho_{\varepsilon} d x \leq \frac{1}{\log R} \int_{\left\{\rho_{\varepsilon} \geq R\right\}} \rho_{\varepsilon} \log \rho_{\varepsilon} d x \leq \frac{C}{\log R}
$$

for any $R>1$ that $\left(\rho_{\varepsilon}\right)$ is equi-integrable. Vitali's convergence theorem implies that $\rho_{\varepsilon} \rightarrow \rho$ strongly in $L^{1}(\Omega)$. Furthermore, possibly for a subsequence, $\sqrt{\rho_{\varepsilon} \theta_{\varepsilon}} \rightarrow \sqrt{\rho \theta}$ a.e. in $\Omega$. The $H^{1}(\Omega)$ bound for $\left(\sqrt{\rho_{\varepsilon} \theta_{\varepsilon}}\right)$ then yields

$\sqrt{\rho_{\varepsilon} \theta_{\varepsilon}} \rightarrow \sqrt{\rho \theta}$ strongly in $L^{p}(\Omega)$ and weakly in $H^{1}(\Omega)$ and $L^{6}(\Omega)$,

where $1<p<6$. Furthermore, we have

$$
\begin{array}{r}
E_{\varepsilon}=\left(1+\frac{3}{2} \rho_{\varepsilon}\right) \theta_{\varepsilon} \rightarrow E:=\left(1+\frac{3}{2} \rho\right) \theta \quad \text { weakly in } L^{3}(\Omega), \\
\nabla\left(\rho_{\varepsilon} \theta_{\varepsilon}\right)=2 \sqrt{\rho_{\varepsilon} \theta_{\varepsilon}} \nabla \sqrt{\rho_{\varepsilon} \theta_{\varepsilon}} \rightarrow \nabla(\rho \theta) \quad \text { weakly in } L^{3 / 2}(\Omega), \\
\nabla\left(\rho_{\varepsilon} \theta_{\varepsilon}^{2}\right)=\rho_{\varepsilon} \theta_{\varepsilon} \nabla \theta_{\varepsilon}+\theta_{\varepsilon} \nabla\left(\rho_{\varepsilon} \theta_{\varepsilon}\right) \rightarrow \nabla\left(\rho \theta^{2}\right) \quad \text { weakly in } L^{6 / 5}(\Omega) .
\end{array}
$$

We deduce from the strong convergence of $\left(\phi_{\varepsilon}\right),\left(\rho_{\varepsilon}\right)$, and $\left(\theta_{\varepsilon}\right)$ as well as from the a.e. positivity of $\rho$ and $\theta$ that $\phi=\log \rho-\frac{3}{2} \log \theta+\frac{5}{2}$ a.e. in $\Omega$.

The uniform bounds for $w_{\varepsilon}$ are sufficient to pass to the limit $\varepsilon \rightarrow 0$ in the $\varepsilon$-terms,

$$
\begin{aligned}
\varepsilon D^{2} \phi_{\varepsilon} \rightarrow 0 & \text { strongly in } L^{2}(\Omega), \\
\varepsilon \theta_{\varepsilon} D^{2} w_{\varepsilon} \rightarrow 0 & \text { strongly in } L^{1}(\Omega), \\
\varepsilon \theta_{\varepsilon}\left|\nabla w_{\varepsilon}\right|^{2} \nabla w_{\varepsilon} \rightarrow 0 & \text { strongly in } L^{1}(\Omega), \\
\varepsilon\left(1+\theta_{\varepsilon}\right) w_{\varepsilon} \rightarrow 0 & \text { strongly in } L^{2}(\Omega),
\end{aligned}
$$

as well as in the $\delta$-terms. The most difficult term is $\delta \int_{\Omega} e^{-N w_{\varepsilon}} w_{\varepsilon} \psi_{2} d x$. It follows from $\sqrt{\theta}|\log \theta|^{(2 N+1) /(2 N)} \leq C$ for $\theta \leq 1$ and $\theta^{-(N+1)} \sqrt{\theta}|\log \theta|^{(2 N+1) /(2 N)} \leq$ 
$C$ for $\theta>1$ as well as from (24) that

$$
\begin{aligned}
\delta\left\|e^{-N w_{\varepsilon}} w_{\varepsilon}\right\|_{L^{(2 N+1) /(2 N)}(\Omega)}^{(2 N+1) /(2 N)} & =\delta \int_{\Omega} \theta_{\varepsilon}^{-(N+1)} \sqrt{\theta_{\varepsilon}}\left|\log \theta_{\varepsilon}\right|^{(2 N+1) /(2 N)} d x \\
& \leq \delta \int_{\Omega} \theta_{\varepsilon}^{-(N+1)} d x+\delta C \leq C(\tau) .
\end{aligned}
$$

Since $\delta e^{-N w_{\varepsilon}} w_{\varepsilon} \rightarrow \delta \theta^{-N} \log \theta$ a.e. in $\Omega$, we conclude that this limit also holds strongly in $L^{1}(\Omega)$. Therefore, we can perform the limit $\varepsilon \rightarrow 0$ in (21)-(22) (now writing the superindex $k$ ) leading to

$$
\begin{aligned}
0= & \frac{1}{\tau} \int_{\Omega}\left(\rho^{k}-\rho^{k-1}\right) \psi_{1} d x+\int_{\Omega} \nabla\left(\rho^{k} \theta^{k}\right) \cdot \nabla \psi_{1} d x+\delta \int_{\Omega}\left(\nabla \phi^{k} \cdot \nabla \psi_{1}+\phi^{k} \psi_{1}\right) d x \\
0= & \frac{1}{\tau} \int_{\Omega}\left(E^{k}-E^{k-1}\right) \psi_{2} d x+\int_{\Omega} \nabla\left(\theta^{k}+\frac{5}{2} \rho^{k}\left(\theta^{k}\right)^{2}\right) \cdot \nabla \psi_{2} d x \\
& +\delta \int_{\Omega}\left(\theta^{k}\right)^{2} \nabla \theta^{k} \cdot \nabla \psi_{2} d x+\delta \int_{\Omega}\left(\theta^{k}\right)^{-N} \log \left(\theta^{k}\right) \psi_{2} d x
\end{aligned}
$$

for any test functions $\psi_{1} \in W^{1,3}(\Omega), \psi_{2} \in W^{1,6}(\Omega)$.

\subsection{Limit $\tau \rightarrow 0$}

We introduce the piecewise constant functions in time $\rho_{\tau}(x, t)=\rho^{k}(x), \theta_{\tau}(x, t)$ $=\theta^{k}(x), \phi_{\tau}(x, t)=\phi^{k}(x)$, and $E_{\tau}(x, t)=E^{k}(x)$ for $x \in \Omega, t \in((k-1) \tau, k \tau]$. Furthermore, let $\left(\pi_{\tau} u\right)(x, t)=u^{k-1}(x)$ for $x \in \Omega, t \in((k-1) \tau, k \tau]$ be the shift operator for piecewise constant functions $u$. We reformulate (26)-(27):

$$
\begin{aligned}
0= & \frac{1}{\tau} \int_{0}^{T} \int_{\Omega}\left(\rho_{\tau}-\pi_{\tau} \rho_{\tau}\right) \psi_{1} d x d t+\int_{0}^{T} \int_{\Omega} \nabla\left(\rho_{\tau} \theta_{\tau}\right) \cdot \nabla \psi_{1} d x d t \\
& +\delta \int_{0}^{T} \int_{\Omega}\left(\nabla \phi_{\tau} \cdot \nabla \psi_{1}+\phi_{\tau} \psi_{1}\right) d x d t \\
0= & \frac{1}{\tau} \int_{0}^{T} \int_{\Omega}\left(E_{\tau}-\pi_{\tau} E_{\tau}\right) \psi_{2} d x d t+\int_{0}^{T} \int_{\Omega} \nabla\left(\theta_{\tau}+\frac{5}{2} \rho_{\tau} \theta_{\tau}^{2}\right) \cdot \nabla \psi_{2} d x d t \\
& +\delta \int_{0}^{T} \int_{\Omega} \theta_{\tau}^{2} \nabla \theta_{\tau} \cdot \nabla \psi_{2} d x d t+\delta \int_{0}^{T} \int_{\Omega} \theta_{\tau}^{-N} \log \left(\theta_{\tau}\right) \psi_{2} d x d t
\end{aligned}
$$

for piecewise constant test functions in time $\psi_{1}, \psi_{2} \in L^{2}\left(0, T ; W^{1,6}(\Omega)\right)$. By density [16, Prop. 1.36], these formulations hold for all test functions in $L^{2}\left(0, T ; W^{1,6}(\Omega)\right)$. We collect the uniform estimates from the discrete entropy inequality $(23)$ :

$$
\begin{array}{r}
\left\|\rho_{\tau} \log \rho_{\tau}\right\|_{L^{\infty}\left(0, T ; L^{1}(\Omega)\right)}+\left\|\theta_{\tau}\right\|_{L^{\infty}\left(0, T ; L^{1}(\Omega)\right)} \leq C, \\
\left\|\rho_{\tau} \theta_{\tau}\right\|_{L^{\infty}\left(0, T ; L^{1}(\Omega)\right)}+\left\|\sqrt{\theta_{\tau}} \nabla \sqrt{\rho_{\tau}}\right\|_{L^{2}\left(\Omega_{T}\right)}+\left\|\sqrt{\rho_{\tau} \theta_{\tau}}\right\|_{L^{2}\left(0, T ; H^{1}(\Omega)\right)} \leq C, \\
\left\|\log \theta_{\tau}\right\|_{L^{2}\left(0, T ; H^{1}(\Omega)\right)}+\left\|\log \theta_{\tau}\right\|_{L^{\infty}\left(0, T ; L^{1}(\Omega)\right)} \leq C, \\
\sqrt{\delta}\left\|\phi_{\tau}\right\|_{L^{2}\left(0, T ; H^{1}(\Omega)\right)}+\sqrt{\delta}\left\|\nabla \theta_{\tau}\right\|_{L^{2}\left(\Omega_{T}\right)}+\delta\left\|\theta_{\tau}^{-(N+1)}\right\|_{L^{1}\left(\Omega_{T}\right)} \leq C,
\end{array}
$$

where the constant $C>0$ does not depend on $\tau$ or $\delta$. In the following, we show some additional estimates for $\left(\rho_{\tau}, \theta_{\tau}\right)$. 
Lemma 2. (Mass and energy control). It holds for any $t \in(0, T)$ that

$$
\left|\int_{\Omega} \rho_{\tau}(t) d x-\int_{\Omega} \rho^{0} d x\right| \leq C \delta^{1 / 2}, \quad\left|\int_{\Omega} E_{\tau}(t) d x-\int_{\Omega} E^{0} d x\right| \leq C \delta^{1 /(2 N+1)} .
$$

Proof. Using $\psi_{1}=1$ in (26) and summing from $k=1, \ldots, n$ gives

$$
\int_{\Omega}\left(\rho^{n}-\rho^{0}\right) d x=\sum_{k=1}^{n} \int_{\Omega}\left(\rho_{\tau}^{k}-\rho^{k-1}\right) d x=\tau \delta \sum_{k=1}^{n} \int_{\Omega} \phi^{k} d x,
$$

where $n \leq N$. We infer from bound (33) for $\left(\phi_{\tau}\right)$ that

$$
\left|\int_{\Omega}\left(\rho_{\tau}(t)-\rho^{0}\right) d x\right|=\delta\left|\int_{0}^{t} \int_{\Omega} \phi_{\tau} d x d t\right| \leq \delta^{1 / 2} C .
$$

The second statement follows after choosing $\psi_{2}=1$ in (27) and using (25).

Lemma 3. (Higher integrability). It holds that

$$
\left\|\theta_{\tau}\right\|_{L^{2}\left(\Omega_{T}\right)}+\left\|\rho_{\tau}^{\alpha} \theta_{\tau}^{\beta}\right\|_{L^{1}\left(\Omega_{T}\right)}+\delta^{1 / 4}\left\|\theta_{\tau}\right\|_{L^{4}\left(\Omega_{T}\right)} \leq C,
$$

where $(\alpha, \beta) \in\left\{(1,2),(1,3),\left(\frac{3}{2}, 3\right),(2,1),(2,2),(2,3)\right\}$.

Proof. The proof is based on the $H^{-1}(\Omega)$ method, i.e., we use test functions of the type $(-\Delta)^{-1} \rho_{\tau}$ and $(-\Delta)^{-1} E_{\tau}$. More precisely, let $\Psi_{1}, \Psi_{2} \in$ $L^{\infty}\left(0, T ; H^{1}(\Omega)\right)$ be the unique solutions to, respectively,

$$
\begin{aligned}
& -\Delta \Psi_{1}=\rho_{\tau}-f_{\Omega} \rho_{\tau} d x \quad \text { on } \Omega, \quad \nabla \Psi_{1} \cdot \nu=0 \quad \text { on } \partial \Omega, \quad \int_{\Omega} \Psi_{1} d x=0, \\
& -\Delta \Psi_{2}=E_{\tau}-f_{\Omega} E_{\tau} d x \quad \text { on } \Omega, \quad \nabla \Psi_{2} \cdot \nu=0 \quad \text { on } \partial \Omega, \quad \int_{\Omega} \Psi_{2} d x=0,
\end{aligned}
$$

where $f u d x=\operatorname{meas}(\Omega)^{-1} \int_{\Omega} u d x$.

Step 1: uniform bounds for $\Psi_{2}$. We use the test function $\Psi_{2}$ in the weak formulation of the second equation in (34) and take into account the energy control. Then

$$
\left\|\nabla \Psi_{2}\right\|_{L^{2}(\Omega)}^{2} \leq C\left(1+\left\|E_{\tau}\right\|_{L^{6 / 5}(\Omega)}\right)\left\|\Psi_{2}\right\|_{L^{6}(\Omega)} .
$$

It follows from Sobolev's embedding and the Poincaré-Wirtinger inequality that

$$
\left\|\nabla \Psi_{2}\right\|_{L^{2}(\Omega)}^{2} \leq C\left(1+\left\|E_{\tau}\right\|_{L^{6 / 5}(\Omega)}\right)\left\|\nabla \Psi_{2}\right\|_{L^{2}(\Omega)}
$$

and so

$$
\left\|\Psi_{2}\right\|_{H^{1}(\Omega)} \leq C\left(1+\left\|E_{\tau}\right\|_{L^{6 / 5}(\Omega)}\right) .
$$

We proceed by bootstrapping this result. Elliptic regularity for

$$
-\Delta \Psi_{2}+\Psi_{2}=E_{\tau}-f_{\Omega} E_{\tau} d x+\Psi_{2} \quad \text { in } \Omega
$$

gives (here, we need the boundary regularity $\partial \Omega \in C^{1,1}$ )

$$
\left\|\Psi_{2}\right\|_{W^{2,6 / 5}(\Omega)} \leq C\left(1+\left\|E_{\tau}\right\|_{L^{6 / 5}(\Omega)}+\left\|\Psi_{2}\right\|_{L^{6 / 5}(\Omega)}\right) \leq C\left(1+\left\|E_{\tau}\right\|_{L^{6 / 5}(\Omega)}\right) .
$$


Since $\left(E_{\tau}\right)$ is bounded in $L^{\infty}\left(0, T ; L^{1}(\Omega)\right)$, an interpolation shows that

$$
\begin{aligned}
\left\|E_{\tau}\right\|_{L^{6}\left(0, T ; L^{6 / 5}(\Omega)\right)}^{6} & \leq \int_{0}^{T}\left\|E_{\tau}\right\|_{L^{2}(\Omega)}^{2}\left\|E_{\tau}\right\|_{L^{1}(\Omega)}^{4} d t \\
& \leq\left\|E_{\tau}\right\|_{L^{\infty}\left(0, T ; L^{1}(\Omega)\right)}^{4} \int_{0}^{T}\left\|E_{\tau}\right\|_{L^{2}(\Omega)}^{2} d t .
\end{aligned}
$$

We deduce from the embedding $L^{6}\left(0, T ; W^{2,6 / 5}(\Omega)\right) \hookrightarrow L^{6}\left(\Omega_{T}\right)$ that

$$
\left\|\Psi_{2}\right\|_{L^{6}\left(\Omega_{T}\right)} \leq C\left(1+\left\|E_{\tau}\right\|_{L^{2}\left(\Omega_{T}\right)}^{1 / 3}\right) .
$$

Step 2: Test functions $\Psi_{1}$ and $\Psi_{2}$. We choose $\Psi_{1}$ and $\Psi_{2}$ as test functions in (28) and (29), respectively:

$$
\begin{aligned}
0= & \frac{1}{\tau} \int_{0}^{T} \int_{\Omega}\left(\rho_{\tau}-\pi_{\tau} \rho_{\tau}\right) \Psi_{1} d x d t+\int_{0}^{T} \int_{\Omega} \rho_{\tau} \theta_{\tau}\left(\rho_{\tau}-f_{\Omega} \rho_{\tau} d x\right) d x d t \\
& +\delta \int_{0}^{T} \int_{\Omega} \phi_{\tau}\left(\rho_{\tau}-f_{\Omega} \rho_{\tau} d x+\Psi_{1}\right) d x d t \\
0= & \frac{1}{\tau} \int_{0}^{T} \int_{\Omega}\left(E_{\tau}-\pi_{\tau} E_{\tau}\right) \Psi_{2} d x d t+\int_{0}^{T} \int_{\Omega}\left(\theta_{\tau}+\frac{5}{2} \rho_{\tau} \theta_{\tau}^{2}\right)\left(E_{\tau}-f_{\Omega} E_{\tau} d x\right) d x d t \\
& +\frac{\delta}{3} \int_{0}^{T} \int_{\Omega} \theta_{\tau}^{3}\left(E_{\tau}-f_{\Omega} E_{\tau} d x\right) d x d t+\delta \int_{0}^{T} \int_{\Omega} \theta_{\tau}^{-N} \log \left(\theta_{\tau}\right) \Psi_{2} d x d t .
\end{aligned}
$$

We estimate the first integral in (36). Since $\Psi_{1}$ has zero spatial average and $\nabla \Psi_{1} \cdot \nu=0$ on $\partial \Omega$, it follows from (34) that

$$
\begin{aligned}
\frac{1}{\tau} \int_{0}^{T} \int_{\Omega}\left(\rho_{\tau}-\pi_{\tau} \rho_{\tau}\right) \Psi_{1} d x d t= & \frac{1}{\tau} \int_{0}^{T} \int_{\Omega}\left(\mathrm{id}-\pi_{\tau}\right)\left(\rho_{\tau}-f_{\Omega} \rho_{\tau} d x\right) \Psi_{1} d x d t \\
& +\frac{1}{\tau} \int_{0}^{T}\left(\mathrm{id}-\pi_{\tau}\right)\left(f_{\Omega} \rho_{\tau} d x\right)\left(\int_{\Omega} \Psi_{1} d x\right) d t \\
& =\frac{1}{\tau} \int_{0}^{T} \int_{\Omega} \nabla\left(\left(\mathrm{id}-\pi_{\tau}\right) \Psi_{1}\right) \cdot \nabla \Psi_{1} d x d t .
\end{aligned}
$$

The function $\Psi_{1}$ is piecewise constant in time. We write $\Psi_{1}(x, t)=\Psi_{1}^{k}(x)$ for $x \in \Omega, t \in((k-1) \tau, k \tau]$. Then, using Young's inequality,

$$
\begin{aligned}
\frac{1}{\tau} \int_{0}^{T} & \int_{\Omega} \nabla\left(\left(\mathrm{id}-\pi_{\tau}\right) \Psi_{1}\right) \cdot \nabla \Psi_{1} d x d t=\sum_{k=1}^{N} \int_{\Omega} \nabla\left(\Psi_{1}^{k}-\Psi_{1}^{k-1}\right) \cdot \nabla \Psi_{1}^{k} d x \\
\geq & \frac{1}{2} \sum_{k=1}^{N} \int_{\Omega}\left(\left|\nabla \Psi_{1}^{k}\right|^{2}-\left|\nabla \Psi_{1}^{k-1}\right|^{2}\right) d x=\frac{1}{2} \int_{\Omega}\left(\left|\nabla \Psi_{1}^{N}\right|^{2}-\left|\nabla \Psi_{1}^{0}\right|^{2}\right) d x .
\end{aligned}
$$

We conclude that

$$
\frac{1}{\tau} \int_{0}^{T} \int_{\Omega}\left(\rho_{\tau}-\pi_{\tau} \rho_{\tau}\right) \Psi_{1} d x d t \geq \frac{1}{2} \int_{\Omega}\left|\nabla \Psi_{1}(T)\right|^{2} d x-\frac{1}{2} \int_{\Omega}\left|\nabla \Psi_{1}(0)\right|^{2} d x .
$$

In a similar way, we have

$$
\frac{1}{\tau} \int_{0}^{T} \int_{\Omega}\left(E_{\tau}-\pi_{\tau} E_{\tau}\right) \Psi_{2} d x d t \geq \frac{1}{2} \int_{\Omega}\left|\nabla \Psi_{2}(T)\right|^{2} d x-\frac{1}{2} \int_{\Omega}\left|\nabla \Psi_{2}(0)\right|^{2} d x .
$$


Inserting these inequalities into (36) and (37), respectively, and adding both inequalities, we find that

$$
\begin{aligned}
\frac{1}{2} \int_{\Omega}\left|\nabla \Psi_{1}(T)\right|^{2} d x+\frac{1}{2} \int_{\Omega}\left|\nabla \Psi_{2}(T)\right|^{2} d x+\frac{\delta}{3} \int_{0}^{T} \int_{\Omega} \theta_{\tau}^{3} E_{\tau} d x d t \\
\quad+\int_{0}^{T} \int_{\Omega} \rho_{\tau}^{2} \theta_{\tau} d x d t+\int_{0}^{T} \int_{\Omega}\left(\theta_{\tau}+\frac{5}{2} \rho_{\tau} \theta_{\tau}^{2}\right)\left(\theta_{\tau}+\frac{3}{2} \rho_{\tau} \theta_{\tau}\right) d x d t \\
\leq \frac{1}{2} \int_{\Omega}\left|\nabla \Psi_{1}(0)\right|^{2} d x+\frac{1}{2} \int_{\Omega}\left|\nabla \Psi_{2}(0)\right|^{2} d x-\delta \int_{0}^{T} \int_{\Omega} \phi_{\tau}\left(\rho_{\tau}-f_{\Omega} \rho_{\tau} d x+\Psi_{1}\right) d x d t \\
\quad+\frac{3}{2} \int_{0}^{T}\left(\int_{\Omega} \rho_{\tau} \theta_{\tau} d x\right)\left(f_{\Omega} \rho_{\tau} d x\right) d t+\int_{0}^{T} \int_{\Omega}\left(\theta_{\tau}+\frac{5}{2} \rho_{\tau} \theta_{\tau}^{2}\right) d x\left(f_{\Omega} E_{\tau} d x\right) d t \\
\quad+\frac{\delta}{3} \int_{0}^{T} \int_{\Omega} \theta_{\tau}^{3}\left(f_{\Omega} E_{\tau} d x\right) d x d t-\delta \int_{0}^{T} \int_{\Omega} \theta_{\tau}^{-N} \log \left(\theta_{\tau}\right) \Psi_{2} d x d t \\
=: J_{1}+\cdots+J_{7} .
\end{aligned}
$$

We start with the last integral. It follows from (35) that

$J_{7} \leq \delta\left\|\theta_{\tau}^{-N} \log \theta_{\tau}\right\|_{L^{6 / 5}\left(\Omega_{T}\right)}\left\|\Psi_{2}\right\|_{L^{6}\left(\Omega_{T}\right)} \leq \delta C\left\|\theta_{\tau}^{-N} \log \theta_{\tau}\right\|_{L^{6 / 5}\left(\Omega_{T}\right)}\left(1+\left\|E_{\tau}\right\|_{L^{2}\left(\Omega_{T}\right)}^{1 / 3}\right)$.

The first norm is estimated according to

$$
\begin{aligned}
\left\|\theta_{\tau}^{-N} \log \theta_{\tau}\right\|_{L^{6 / 5}\left(\Omega_{T}\right)}^{6 / 5} & =\int_{0}^{T} \int_{\Omega} \theta_{\tau}^{-6 N / 5}\left|\log \theta_{\tau}\right|^{6 / 5} d x d t \\
& \leq C+\int_{0}^{T} \int_{\Omega \cap\left\{\theta_{\tau}(t)<1\right\}} \theta_{\tau}^{-6 N / 5}\left|\log \theta_{\tau}\right|^{6 / 5} d x d t \\
& \leq C+C \int_{0}^{T} \int_{\Omega \cap\left\{\theta_{\tau}(t)<1\right\}}^{\theta_{\tau}^{-(N+1)} d x d t}
\end{aligned}
$$

where the last inequality follows from the condition $N<5$ (and hence $6 N / 5<$ $N+1)$. Because of (33), this leads to

$$
\delta\left\|\theta_{\tau}^{-N} \log \theta_{\tau}\right\|_{L^{6 / 5}\left(\Omega_{T}\right)} \leq C \delta^{1 / 6} .
$$

Therefore, we infer that

$$
J_{7} \leq \delta^{1 / 6} C\left(1+\left\|E_{\tau}\right\|_{L^{2}\left(\Omega_{T}\right)}^{1 / 3}\right) .
$$

Since $E_{\tau}=\theta_{\tau}+\frac{3}{2} \rho_{\tau} \theta_{\tau}$, the right-hand side can be controlled (for sufficiently small $\delta>0$ ) by the last two integrals on the left-hand side of (38).

Next, we consider the following term appearing in $J_{3}$ :

$$
\begin{aligned}
-\delta \int_{0}^{T} \int_{\Omega} \phi \rho_{\tau} d x d t & =-\delta \int_{0}^{T} \int_{\Omega}\left(\log \left(\rho_{\tau} \theta_{\tau}^{-3 / 2}\right)+\frac{5}{2}\right) \rho_{\tau} d x d t \\
& \leq-\delta \int_{0}^{T} \int_{\Omega} \theta_{\tau}^{3 / 2} \cdot \rho_{\tau} \theta_{\tau}^{-3 / 2} \log \left(\rho_{\tau} \theta_{\tau}^{-3 / 2}\right) d x d t+C \\
& \leq \delta C \int_{0}^{T} \int_{\Omega} \theta_{\tau}^{3 / 2} d x d t+C
\end{aligned}
$$


where the last inequality follows from the fact that $z \mapsto z \log z$ is bounded from below. Furthermore, we deduce from Lemma 2, bound (33) for $\phi_{\tau}$, and the Poincaré-Wirtinger inequality that

$$
\begin{aligned}
\delta \int_{0}^{T} \int_{\Omega} \phi_{\tau}\left(f_{\Omega} \rho_{\tau} d x\right) d x d t & \leq \delta\left\|\phi_{\tau}\right\|_{L^{1}\left(\Omega_{T}\right)}\left\|\rho_{\tau}\right\|_{L^{\infty}\left(0, T ; L^{1}(\Omega)\right)} \leq C \\
\delta \int_{0}^{T} \int_{\Omega} \phi_{\tau} \Psi_{1} d x d t & \leq \frac{\delta}{2} \int_{0}^{T} \int_{\Omega} \phi_{\tau}^{2} d x d t+\frac{\delta}{2} \int_{\Omega} \Psi_{1}^{2} d x d t \\
& \leq C+\delta C \int_{0}^{T} \int_{\Omega}\left|\nabla \Psi_{1}\right|^{2} d x d t
\end{aligned}
$$

This shows that

$$
J_{3} \leq C+\delta C \int_{0}^{T} \int_{\Omega} \theta_{\tau}^{3 / 2} d x d t+\delta C \int_{0}^{T} \int_{\Omega}\left|\nabla \Psi_{1}\right|^{2} d x d t .
$$

The first integral on the right-hand side can be controlled by the last integral on the left-hand side of (38). The last integral on the right-hand side is controlled after applying Gronwall's inequality. The integrals $J_{4}, J_{5}$, and $J_{6}$ can be controlled by the expressions on the left-hand side of (38). We conclude that

$$
\begin{aligned}
& \int_{\Omega}\left(\left|\nabla \Psi_{1}(T)\right|^{2}+\left|\nabla \Psi_{2}(T)\right|^{2}\right) d x \\
& \quad+\int_{0}^{T} \int_{\Omega}\left(\theta_{\tau}^{2}+\delta \theta_{\tau}^{4}+\rho_{\tau} \theta_{\tau}^{2}\left(1+\theta_{\tau}\right)+\rho_{\tau}^{2} \theta_{\tau}\left(1+\theta_{\tau}^{2}\right)\right) d x d t \leq C \exp (\delta C T) .
\end{aligned}
$$

We deduce from this estimate and Young's inequality that

$$
\begin{aligned}
\left\|\rho_{\tau} \theta_{\tau}\right\|_{L^{2}\left(\Omega_{T}\right)}^{2} & \leq \frac{1}{2} \int_{0}^{T} \int_{\Omega} \rho_{\tau}^{2}\left(\theta_{\tau}+\theta_{\tau}^{3}\right) d x d t \leq C, \\
\left\|\rho_{\tau} \theta_{\tau}^{2}\right\|_{L^{3 / 2}\left(\Omega_{T}\right)}^{3 / 2} & \leq \frac{1}{2} \int_{0}^{T} \int_{\Omega}\left(\rho_{\tau}+\rho_{\theta}^{2}\right) \theta_{\tau}^{3} d x d t \leq C .
\end{aligned}
$$

This proves the lemma.

Step 3: Strong convergence of $\left(\rho_{\tau}\right)$ and $\left(\theta_{\tau}\right)$. First, we prove a gradient bound for the particle density.

Lemma 4. (Gradient estimate). There exist $N \in(0,5), m \in\left(\frac{1}{2}, 1\right)$, and $\alpha \in$ $\left(\frac{2}{3}, 1\right)$ such that

$$
\left\|\rho_{\tau}^{m}\right\|_{L^{p}\left(0, T ; W^{1, q}(\Omega)\right)} \leq C(\delta),
$$

where $C(\delta)>0$ does not depend on $\tau, p \geq 1 / m$, and $3 q /(3-q)>1 / m$ (or equivalently, $q>3 /(3 m+1))$. Moreover, with a constant $C>0$ independent of $\tau$ and $\delta$,

$$
\left\|E_{\tau}\right\|_{L^{1}\left(0, T ; W^{1,1}(\Omega)\right)} \leq C .
$$

The condition $q>3 /(3 m+1)$ guarantees that $W^{1, q}(\Omega) \hookrightarrow L^{1 / m}(\Omega)$. This is needed below for the application of the nonlinear Aubin-Lions lemma. 
Proof. It follows from Lemma 3 that $\left(\rho_{\tau} \theta_{\tau}^{1 / 2}\right)$ is bounded in $L^{2}\left(\Omega_{T}\right)$, while estimate (33) implies that $\left(\theta_{\tau}^{-1 / 2}\right)$ is bounded in $L^{2(N+1)}\left(\Omega_{T}\right)$. Consequently, $\rho_{\tau}=\rho_{\tau} \theta^{1 / 2} \theta_{\tau}^{-1 / 2}$ is uniformly bounded in $L^{r}\left(\Omega_{T}\right)$, where $r:=2(N+1) /(N+$ $2)>1$. Together with the $L^{\infty}\left(0, T ; L^{1}(\Omega)\right)$ bound for $\left(\rho_{\tau}\right)$, an interpolation with $1 / c=(1-\alpha) / 1+\alpha / r$ and $b \geq 1$ gives

$$
\left\|\rho_{\tau}\right\|_{L^{b}\left(0, T ; L^{c}(\Omega)\right)}^{b} \leq\left\|\rho_{\tau}\right\|_{L^{\infty}\left(0, T ; L^{1}(\Omega)\right)}^{(1-\alpha) b} \int_{0}^{T}\left\|\rho_{\tau}\right\|_{L^{r}(\Omega)}^{\alpha b} d t \leq C \int_{0}^{T}\left\|\rho_{\tau}\right\|_{L^{r}(\Omega)}^{\alpha b} d t .
$$

A simple computation shows that $c=r /(\alpha+(1-\alpha) r)$. We choose $b=r / \alpha$ and use the $L^{r}\left(\Omega_{T}\right)$ bound for $\left(\rho_{\tau}\right)$ :

$$
\left\|\rho_{\tau}\right\|_{L^{r / \alpha}\left(0, T ; L^{r /(\alpha+(1-\alpha) r)}(\Omega)\right)} \leq C \quad \text { for } r=\frac{2(N+1)}{N+2}, \alpha \in(0,1) .
$$

Let $\frac{1}{2}<m<1$. Then

$$
\left\|\rho_{\tau}^{m}\right\|_{L^{r /(\alpha m)}\left(0, T ; L^{r /(m(\alpha+(1-\alpha) r))(\Omega))}\right.} \leq C .
$$

We know from (32) and (33) that $\nabla \log \rho_{\tau}=\nabla \phi_{\tau}+\frac{3}{2} \nabla \log \theta_{\tau}$ is uniformly bounded in $L^{2}\left(\Omega_{T}\right)$ (but not uniformly in $\delta$ ). It follows that $\nabla \rho_{\tau}^{m}=m \rho_{\tau}^{m} \nabla \log \rho_{\tau}$ is uniformly bounded in $L^{p}\left(0, T ; L^{q}(\Omega)\right)$, where $p, q \geq 1$ satisfy

$$
\frac{1}{p}=\frac{1}{2}+\frac{\alpha m}{r}, \quad \frac{1}{q}=\frac{1}{2}+\frac{m}{r}(\alpha+(1-\alpha) r) .
$$

We deduce from the Poincaré-Wirtinger inequality and the $L^{\infty}\left(0, T ; L^{1}(\Omega)\right)$ bound for $\left(\rho_{\tau}\right)$ that

$$
\left\|\rho_{\tau}^{m}\right\|_{L^{p}\left(0, T ; L^{q}(\Omega)\right)} \leq C\left\|\nabla \rho_{\tau}^{m}\right\|_{L^{p}\left(0, T ; L^{q}(\Omega)\right)}+C\left\|\rho_{\tau}^{m}\right\|_{L^{p}\left(0, T ; L^{1}(\Omega)\right)} \leq C(\delta) .
$$
that

We claim that there exist $N \in(0,5), m \in\left(\frac{1}{2}, 1\right)$, and $\alpha \in(0,1)$ such

$$
p \geq \frac{1}{m}, \quad \frac{3 q}{3-q}>\frac{1}{m},
$$

where $p$ and $q$ are given by (40). A straightforward computation shows that these inequalities are equivalent to

$$
r \geq \frac{2 \alpha m}{2 m-1}, \quad \frac{r}{r-1}<6 \alpha m
$$

We choose $r=2 \alpha m /(2 m-1)$ (recall that $m>1 / 2)$ such that the first inequality is satisfied. With this choice, the second inequality is equivalent to $m<1 /(3(1-\alpha))$. Since we want $m<1$, we need to choose $\alpha>2 / 3$. Then $\frac{1}{2}<m<1<1 /(3(1-\alpha))$. By definition of $r$,

$$
\frac{2(N+1)}{N+2}=r=\frac{2 \alpha m}{2 m-1} .
$$

Thus, it remains to prove that $N \in(0,5)$ can be chosen such that this identity holds for some $\alpha>\frac{2}{3}$ and $m \in\left(\frac{1}{2}, 1\right)$. Equation (41) is equivalent to

$$
N=-\frac{2 \alpha m-2 m+1}{\alpha m-2 m+1},
$$


and the requirement $N<5$ gives $m>6 /(12-7 \alpha)$. The right-hand side is smaller than one if $\alpha<\frac{6}{7}$. This is compatible with the previous constraint $\alpha>\frac{2}{3}$ and proves the claim.

To finish the proof of the lemma, we observe that (31) and Lemma 3 imply that

$$
\left\|\nabla\left(\rho_{\tau} \theta_{\tau}\right)\right\|_{L^{4 / 3}\left(\Omega_{T}\right)} \leq 2\left\|\sqrt{\rho_{\tau} \theta_{\tau}}\right\|_{L^{4}\left(\Omega_{T}\right)}\left\|\nabla \sqrt{\rho_{\tau} \theta_{\tau}}\right\|_{L^{2}\left(\Omega_{T}\right)} \leq C .
$$

Moreover, we deduce from (32) and Lemma 3 that

$$
\left\|\nabla \theta_{\tau}\right\|_{L^{1}\left(\Omega_{T}\right)} \leq\left\|\theta_{\tau}\right\|_{L^{2}\left(\Omega_{T}\right)}\left\|\nabla \log \theta_{\tau}\right\|_{L^{2}\left(\Omega_{T}\right)} \leq C .
$$

Thus, $\left(E_{\tau}\right)$ is bounded in $L^{1}\left(0, T ; W^{1,1}(\Omega)\right)$, and the proof is finished.

Lemma 5. (Bounds for the discrete time derivative). There exists a constant $C>0$ which does not depend on $\tau$ such that

$\tau^{-1}\left\|\rho_{\tau}-\pi_{\tau} \rho_{\tau}\right\|_{L^{4 / 3}\left(0, T ; W^{1,4}(\Omega)^{\prime}\right)} \leq C, \quad \tau^{-1}\left\|E_{\tau}-\pi_{\tau} E_{\tau}\right\|_{L^{6 / 5}\left(0, T ; W^{2,4}(\Omega)^{\prime}\right)} \leq C$.

Proof. We infer from (42) and (33) that

$$
\begin{aligned}
\tau^{-1}\left\|\rho_{\tau}-\pi_{\tau} \rho_{\tau}\right\|_{L^{4 / 3}\left(0, T ; W^{1,4}(\Omega)^{\prime}\right)} & =\sup _{\left\|\psi_{1}\right\|_{L^{4}\left(0, T ; W^{1,4}(\Omega)\right)}=1}\left|\tau^{-1} \int_{0}^{T} \int_{\Omega}\left(\rho_{\tau}-\pi_{\tau} \rho_{\tau}\right) \psi_{1} d x d t\right| \\
& \leq \frac{3}{2}\left\|\nabla\left(\rho_{\tau} \theta_{\tau}\right)\right\|_{L^{4 / 3}\left(\Omega_{T}\right)}+\delta\left\|\phi_{\tau}\right\|_{L^{4 / 3}\left(\Omega_{T}\right)} \leq C .
\end{aligned}
$$

Furthermore,

$$
\begin{aligned}
\tau^{-1}\left\|E_{\tau}-\pi_{\tau} E_{\tau}\right\|_{L^{6 / 5}\left(0, T ; W^{2,4}(\Omega)^{\prime}\right)} \leq & \left\|\theta_{\tau}\right\|_{L^{2}\left(\Omega_{T}\right)}+\frac{15}{4}\left\|\rho_{\tau} \theta_{\tau}^{2}\right\|_{L^{3 / 2}\left(\Omega_{T}\right)} \\
& +\frac{\delta}{3}\left\|\theta_{\tau}^{3}\right\|_{L^{4 / 3}\left(\Omega_{T}\right)}+\delta\left\|\theta_{\tau}^{-N} \log \theta_{\tau}\right\|_{L^{6 / 5}\left(\Omega_{T}\right)} .
\end{aligned}
$$

Taking into account Lemma 3, the first three terms on the right-hand side are uniformly bounded. Since $N<5$, the last term can be estimated from above by $\delta\left\|\theta_{\tau}^{-(N+1)}\right\|_{L^{1}\left(\Omega_{T}\right)}^{5 / 6}$ which is bounded because of (33). This finishes the proof.

Lemmas 4 and 5 allow us to apply the Aubin-Lions lemma in the version of [7, Theorem 3]. This is possible since $p \geq 1 / m$ and $W^{1, q}(\Omega) \hookrightarrow L^{1 / m}(\Omega)$ (the last fact is a consequence of $q>3 /(3 m+1)$ ). We infer the existence of a subsequence which is not relabeled such that, as $\tau \rightarrow 0$,

$$
\rho_{\tau} \rightarrow \rho \text { strongly in } L^{1}\left(\Omega_{T}\right) .
$$

Concerning $\left(E_{\tau}\right)$, Lemmas 4 and 5 allow us to apply the Aubin-Lions lemma in the version of [9] (or Theorem 3 in [7] with $m=1$ ) to obtain a subsequence of $\left(E_{\tau}\right)$ (not relabeled) such that, as $\tau \rightarrow 0$,

$$
E_{\tau} \rightarrow E \quad \text { strongly in } L^{1}\left(\Omega_{T}\right)
$$

In fact, because of the $L^{2}\left(\Omega_{T}\right)$ bound for $\left(E_{\tau}\right)$ from Lemma 3 , this convergence holds in $L^{\eta}\left(\Omega_{T}\right)$ for any $\eta<2$. Up to subsequences, we know that $\rho_{\tau} \rightarrow \rho$ and $E_{\tau} \rightarrow E$ a.e. in $\Omega_{T}$. Thus,

$$
\theta_{\tau}=\frac{E_{\tau}}{1+3 \rho_{\tau} / 2} \rightarrow \frac{E}{1+3 \rho / 2}=: \theta \quad \text { a.e. in } \Omega_{T} \text {. }
$$


In particular, $E=\theta+\frac{3}{2} \rho \theta$. The bound for $\left(\theta_{\tau}\right)$ in $L^{4}\left(\Omega_{T}\right)$ (not uniform in $\delta$ ) shows that the previous convergence holds in $L^{\eta}\left(\Omega_{T}\right)$ for any $\eta<4$. We deduce from the $L^{2}\left(\Omega_{T}\right)$ bounds for $\log \theta_{\tau}$ and $\log \rho_{\tau}=\phi_{\tau}+\frac{3}{2} \log \theta_{\tau}-\frac{5}{2}$ that $\log \theta$ and $\log \rho$ are integrable and thus, $\rho>0, \theta>0$ a.e. in $\Omega_{T}$. Furthermore, $\phi_{\tau} \rightarrow \log \rho-\frac{3}{2} \log \theta+\frac{5}{2}=: \phi$ a.e. in $\Omega_{T}$ and, because of (33), weakly in $L^{2}\left(0, T ; H^{1}(\Omega)\right)$.

The previous bounds and the strong convergences of $\left(\rho_{\tau}\right)$ and $\left(\theta_{\tau}\right)$ allow us to pass to the limit $\tau \rightarrow 0$ in (28)-(29). For this, we observe that, by (42),

$$
\nabla\left(\rho_{\tau} \theta_{\tau}\right) \rightarrow \nabla(\rho \theta) \quad \text { weakly in } L^{4 / 3}\left(\Omega_{T}\right) .
$$

Furthermore, by Lemma 3,

$$
\rho_{\tau} \theta_{\tau}^{2} \rightarrow \rho \theta^{2} \quad \text { strongly in } L^{\eta}\left(\Omega_{T}\right), \eta<\frac{3}{2} .
$$

The strong convergence of $\left(\theta_{\tau}\right)$ to $\theta$, the uniform bounds on $\left(\theta_{\tau}\right)$, and the a.e. positivity of $\theta$ imply that

$$
\theta_{\tau}^{3} \rightarrow \theta^{3}, \quad \theta_{\tau}^{-N} \log \theta_{\tau} \rightarrow \theta^{-N} \log \theta \quad \text { strongly in } L^{1}\left(\Omega_{T}\right) .
$$

Finally, by Lemma 5,

$$
\begin{array}{cc}
\tau^{-1}\left(\rho_{\tau}-\pi_{\tau} \rho_{\tau}\right) \rightarrow \partial_{t} \rho & \text { weakly in } L^{4 / 3}\left(0, T ; W^{1,4}(\Omega)^{\prime}\right), \\
\tau^{-1}\left(E_{\tau}-\pi_{\tau} E_{\tau}\right) \rightarrow \partial_{t} E & \text { weakly in } L^{6 / 5}\left(0, T ; W^{2,4}(\Omega)^{\prime}\right) .
\end{array}
$$

Then (28)-(29) become in the limit $\tau \rightarrow 0$,

$$
\begin{aligned}
0= & \int_{0}^{T}\left\langle\partial_{t} \rho, \psi_{1}\right\rangle d t+\int_{0}^{T} \int_{\Omega} \nabla(\rho \theta) \cdot \nabla \psi_{1} d x d t+\delta \int_{0}^{T} \int_{\Omega}\left(\nabla \phi \cdot \nabla \psi_{1}+\phi \psi_{1}\right) d x d t \\
0= & \int_{0}^{T}\left\langle\partial_{t} E, \psi_{2}\right\rangle d t-\int_{0}^{T} \int_{\Omega}\left(\theta+\frac{5}{2} \rho \theta^{2}\right) \Delta \psi_{2} d x d t \\
& -\frac{\delta}{3} \int_{0}^{T} \int_{\Omega} \theta^{3} \Delta \psi_{2} d x d t+\delta \int_{0}^{T} \int_{\Omega} \theta^{-N} \log (\theta) \psi_{2} d x d t
\end{aligned}
$$

for any test functions $\psi_{1}, \psi_{2} \in C_{0}^{2}\left(\Omega_{T}\right)$.

\subsection{Limit $\delta \rightarrow 0$}

In this subsection, we need some tools from mathematical fluid dynamics, in particular the concept of renormalized solutions and the div-curl lemma. In the following, we denote by $\overline{u_{\delta}}$ the weak or distributional limit of a sequence $\left(u_{\delta}\right)$ whenever it exists. Let $\left(\rho_{\delta}, E_{\delta}\right)$ be a weak solution to (43)-(44) and set $\phi_{\delta}=\log \left(\rho_{\delta} / \theta_{\delta}^{3 / 2}\right)+\frac{5}{2}, E_{\delta}=\theta_{\delta}+\frac{3}{2} \rho_{\delta} \theta_{\delta}$.

Step 1: Renormalized mass balance equation. We compute the renormalized form of (43). Let $f \in C^{2}([0, \infty)) \cap L^{\infty}(0, \infty)$ satisfy $\left|f^{\prime}(s)\right| \leq C(1+s)^{-1}$ and $\left|f^{\prime \prime}(s)\right| \leq C(1+s)^{-2}$ for $s \geq 0$. Furthermore, let $\xi \in C_{0}^{\infty}\left(\Omega_{T}\right)$. Choosing 
$\psi_{1}=f^{\prime}\left(\rho_{\delta}\right) \xi$ in $(43)$, we find that

$$
\begin{aligned}
\int_{0}^{T} & \left\langle\partial_{t} f\left(\rho_{\delta}\right), \xi\right\rangle d t+\int_{0}^{T} \int_{\Omega}\left(f^{\prime}\left(\rho_{\delta}\right) \nabla\left(\rho_{\delta} \theta_{\delta}\right)+\delta f^{\prime}\left(\rho_{\delta}\right) \nabla \phi_{\delta}\right) \cdot \nabla \xi d x d t \\
& =-\int_{0}^{T} \int_{\Omega}\left(\delta f^{\prime}\left(\rho_{\delta}\right) \phi_{\delta}+f^{\prime \prime}\left(\rho_{\delta}\right) \nabla \rho_{\delta} \cdot \nabla\left(\rho_{\delta} \theta_{\delta}\right)+\delta f^{\prime \prime}\left(\rho_{\delta}\right) \nabla \rho_{\delta} \cdot \nabla \phi_{\delta}\right) \xi d x d t
\end{aligned}
$$

This computation can be made rigorous (such that $\psi_{1}$ is an admissible test function) by using renormalization techniques; see, e.g., [13, Section 10.18]. The previous equation can be rewritten as

$$
\begin{aligned}
& -\partial_{t} f\left(\rho_{\delta}\right)+\operatorname{div}\left(f^{\prime}\left(\rho_{\delta}\right) \nabla\left(\rho_{\delta} \theta_{\delta}\right)+\delta f^{\prime}\left(\rho_{\delta}\right) \nabla \phi_{\delta}\right) \\
& \quad=\delta f^{\prime}\left(\rho_{\delta}\right) \phi_{\delta}+f^{\prime \prime}\left(\rho_{\delta}\right) \nabla \rho_{\delta} \cdot \nabla\left(\rho_{\delta} \theta_{\delta}\right)+\delta f^{\prime \prime}\left(\rho_{\delta}\right) \nabla \rho_{\delta} \cdot \nabla \phi_{\delta} \quad \text { in } \mathcal{D}^{\prime}\left(\Omega_{T}\right) .
\end{aligned}
$$

Step 2: Application of the div-curl lemma. We apply the div-curl lemma to the vector fields

$$
U_{\delta}=\left(f\left(\rho_{\delta}\right),-f^{\prime}\left(\rho_{\delta}\right) \nabla\left(\rho_{\delta} \theta_{\delta}\right)-\delta f^{\prime}\left(\rho_{\delta}\right) \nabla \phi_{\delta}\right), \quad V_{\delta}=\left(g\left(\theta_{\delta}\right), 0,0,0\right),
$$

where $f$ is as before and $g \in C^{1}([0, \infty)) \cap L^{\infty}(0, \infty)$ satisfies $\left|g^{\prime}(s)\right| \leq C(1+s)^{-1}$ for $s>0$. We know from $(31)$ that $\left(\nabla \sqrt{\rho_{\delta} \theta_{\delta}}\right)$ and $\left(\sqrt{\delta} \nabla \phi_{\delta}\right)$ are bounded in $L^{2}\left(\Omega_{T}\right)$ and from Lemma 3 that $\left(\sqrt{\rho_{\delta} \theta_{\delta}}\right)$ is bounded in $L^{4}\left(\Omega_{T}\right)$. Consequently,

$$
f^{\prime}\left(\rho_{\delta}\right) \nabla\left(\rho_{\delta} \theta_{\delta}\right)+\delta f^{\prime}\left(\rho_{\delta}\right) \nabla \phi_{\delta}=2 f^{\prime}\left(\rho_{\delta}\right) \sqrt{\rho_{\delta} \theta_{\delta}} \nabla \sqrt{\rho_{\delta} \theta_{\delta}}+\delta f^{\prime}\left(\rho_{\delta}\right) \nabla \phi_{\delta}
$$

is uniformly bounded in $L^{4 / 3}\left(\Omega_{T}\right)$. Thus, $\left(U_{\delta}\right)$ is bounded in $L^{4 / 3}\left(\Omega_{T}\right)$. Because of the properties of $g,\left(V_{\delta}\right)$ is trivially bounded in $L^{\infty}\left(\Omega_{T}\right)$.

The left-hand side of (45) equals $-\operatorname{div}_{(t, x)} U_{\delta}$. We wish to bound the right-hand side of (45). For this, we observe that, thanks to (33), the first term $\delta f^{\prime}\left(\rho_{\delta}\right) \phi_{\delta}$ is uniformly bounded in $L^{2}\left(\Omega_{T}\right)$. We rewrite the second term as

$$
f^{\prime \prime}\left(\rho_{\delta}\right) \nabla \rho_{\delta} \cdot \nabla\left(\rho_{\delta} \theta_{\delta}\right)=4 \rho_{\delta} f^{\prime \prime}\left(\rho_{\delta}\right) \sqrt{\theta_{\delta}} \nabla \sqrt{\rho_{\delta}} \cdot \nabla \sqrt{\rho_{\delta} \theta_{\delta}} .
$$

Since $\rho_{\delta}\left|f^{\prime \prime}\left(\rho_{\delta}\right)\right| \leq C \rho_{\delta} /\left(1+\rho_{\delta}\right)^{2} \leq C$ and $\left(\sqrt{\theta_{\delta}} \nabla \sqrt{\rho_{\delta}}\right),\left(\sqrt{\rho_{\delta} \theta_{\delta}}\right)$ are bounded in $L^{2}\left(\Omega_{T}\right)$ by $(31)$, expression $(46)$ is bounded in $L^{1}\left(\Omega_{T}\right)$. In order to bound the last term in (45), we observe that, by (32) and (33),

$$
\sqrt{\delta} \nabla \log \rho_{\delta}=\sqrt{\delta} \nabla \phi_{\delta}+\frac{3}{2} \sqrt{\delta} \nabla \log \theta_{\delta}
$$

is uniformly bounded in $L^{2}\left(\Omega_{T}\right)$. Then

$$
\delta f^{\prime \prime}\left(\rho_{\delta}\right) \nabla \rho_{\delta} \cdot \nabla \phi_{\delta}=f^{\prime \prime}\left(\rho_{\delta}\right) \rho_{\delta}\left(\sqrt{\delta} \nabla \log \rho_{\delta}\right) \cdot\left(\sqrt{\delta} \nabla \phi_{\delta}\right)
$$

is uniformly bounded in $L^{1}\left(\Omega_{T}\right)$. We infer that the right-hand side of (45) and consequently also - $\operatorname{div}_{(t, x)} U_{\delta}$ are uniformly bounded in $L^{1}\left(\Omega_{T}\right)$. By Sobolev's embedding, it follows that $\operatorname{div}_{(t, x)} U_{\delta}$ is relatively compact in $W^{-1, r}\left(\Omega_{T}\right)$ for some $r>1$.

It follows from the uniform $L^{2}\left(\Omega_{T}\right)$ bound for $\left(\nabla \log \theta_{\delta}\right)$ (see $\left.(32)\right)$ and $\theta_{\delta}\left|g^{\prime}\left(\theta_{\delta}\right)\right| \leq C \theta_{\delta} /\left(1+\theta_{\delta}\right) \leq C$ that

$$
\operatorname{curl}_{(t, x)} V_{\delta}=g^{\prime}\left(\theta_{\delta}\right)\left(\begin{array}{cc}
0 & \left(\nabla \theta_{\delta}\right)^{T} \\
\nabla \theta_{\delta} & 0
\end{array}\right)=\theta_{\delta} g^{\prime}\left(\theta_{\delta}\right)\left(\begin{array}{cc}
0 & \left(\nabla \log \theta_{\delta}\right)^{T} \\
\nabla \log \theta_{\delta} & 0
\end{array}\right)
$$


is uniformly bounded in $L^{2}\left(\Omega_{T}\right)$. By Sobolev's embedding, this expression is relatively compact in $W^{-1, r}\left(\Omega_{T} ; \mathbb{R}^{3 \times 3}\right)$ for some $r>1$.

The div-curl lemma [13, Theorem 10.21] implies that $\overline{U_{\delta} \cdot V_{\delta}}=\overline{U_{\delta}} \cdot \overline{V_{\delta}}$ a.e. in $\Omega_{T}$, which means that

$$
\overline{f\left(\rho_{\delta}\right) g\left(\theta_{\delta}\right)}=\overline{f\left(\rho_{\delta}\right)} \overline{g\left(\theta_{\delta}\right)} \quad \text { a.e. in } \Omega_{T}
$$

for all $f \in C^{2}([0, \infty)) \cap L^{\infty}(0, \infty)$ and $g \in C^{1}([0, \infty)) \cap L^{\infty}(0, \infty)$ satisfying $\left|f^{\prime}(s)\right| \leq C(1+s)^{-1},\left|f^{\prime \prime}(s)\right| \leq C(1+s)^{-2}$, and $\left|g^{\prime}(s)\right| \leq C(1+s)^{-1}$ for $s>0$.

Step 3: Proof of $\overline{\rho_{\delta} \theta_{\delta}}=\rho \theta$. We wish to relax the assumptions on the functions $f$ and $g$. To this end, we introduce the truncation function $T_{1} \in$ $C^{2}([0, \infty))$ by $T_{1}(s)=s$ for $0 \leq s<1, T_{1}(s)=2$ for $s>3$, and $T_{1}$ is nondecreasing and concave in $[0, \infty)$. Then we define $T_{k}(s)=k T_{1}(s / k)$ for $s>0$ and $k \in \mathbb{N}$. It is possible to choose $f=T_{k}$ in (47). Together with Fatou's lemma and the boundedness of $g$, we infer that

$$
\begin{aligned}
& \left\|\overline{\rho_{\delta} g\left(\theta_{\delta}\right)}-\overline{\rho_{\delta}} \overline{g\left(\theta_{\delta}\right)}\right\|_{L^{1}\left(\Omega_{T}\right)} \\
& =\left\|\overline{\left(\rho_{\delta}-T_{k}\left(\rho_{\delta}\right)\right) g\left(\theta_{\delta}\right)}-\overline{\left(\rho_{\delta}-T_{k}\left(\rho_{\delta}\right)\right)} \overline{g\left(\theta_{\delta}\right)}\right\|_{L^{1}\left(\Omega_{T}\right)} \\
& \leq C \sup _{0<\delta<1} \int_{\Omega_{T}}\left|T_{k}\left(\rho_{\delta}\right)-\rho_{\delta}\right| d x d t .
\end{aligned}
$$

Furthermore, we deduce from (30) that

$\int_{\Omega_{T}}\left|T_{k}\left(\rho_{\delta}\right)-\rho_{\delta}\right| d x d t \leq C \int_{\left\{\rho_{\delta} \geq k\right\}} \rho_{\delta} d x d t \leq \frac{C}{\log k} \int_{\left\{\rho_{\delta} \geq k\right\}} \rho_{\delta} \log \rho_{\delta} d x d t \leq \frac{C}{\log k}$, such that we obtain for any $k \geq 2$,

$$
\left\|\overline{\rho_{\delta} g\left(\theta_{\delta}\right)}-\overline{\rho_{\delta}} \overline{g\left(\theta_{\delta}\right)}\right\|_{L^{1}\left(\Omega_{T}\right)} \leq \frac{C}{\log k} .
$$

Then the limit $k \rightarrow \infty$ implies that

$$
\overline{\rho_{\delta} g\left(\theta_{\delta}\right)}=\rho \overline{g\left(\theta_{\delta}\right)} \quad \text { a.e. in } \Omega_{T}
$$

for any $g \in C^{1}([0, \infty)) \cap L^{\infty}(0, \infty)$ satisfying $\left|g^{\prime}(s)\right| \leq C(1+s)^{-1}$ for $s>0$. We choose $g=T_{k}$ which leads to

$$
\overline{\rho_{\delta} \theta_{\delta}}-\rho \theta=\overline{\rho_{\delta}\left(\theta_{\delta}-T_{k}\left(\theta_{\delta}\right)\right)}-\rho\left(\theta-\overline{T_{k}\left(\theta_{\delta}\right)}\right) .
$$

We claim that both terms on the right-hand side converge to zero as $k \rightarrow \infty$. Indeed, it follows from Fatou's lemma and the $L^{1}\left(\Omega_{T}\right)$ bound for $\left(\rho_{\delta} \theta_{\delta}^{2}\right)$ from Lemma 3 that

$$
\begin{aligned}
\left\|\overline{\frac{\rho_{\delta}\left(\theta_{\delta}-T_{k}\left(\theta_{\delta}\right)\right)}{1+\rho}}\right\|_{L^{1}\left(\Omega_{T}\right)} & \leq \sup _{0<\delta<1} \int_{\Omega_{T}} \rho_{\delta}\left|\theta_{\delta}-T_{k}\left(\theta_{\delta}\right)\right| d x d t \\
& \leq C \sup _{0<\delta<1} \int_{\left\{\theta_{\delta}>k\right\}} \rho_{\delta} \theta_{\delta} d x d t \\
& \leq \frac{C}{k} \sup _{0<\delta<1} \int_{\left\{\theta_{\delta}>k\right\}} \rho_{\delta} \theta_{\delta}^{2} d x d t \leq \frac{C}{k},
\end{aligned}
$$


while we deduce from Fatou's lemma and the $L^{2}\left(\Omega_{T}\right)$ bound for $\left(\theta_{\delta}\right)$, again from Lemma 3, that

$$
\begin{aligned}
\left\|\frac{\rho\left(\theta-\overline{T_{k}\left(\theta_{\delta}\right)}\right)}{1+\rho}\right\|_{L^{1}\left(\Omega_{T}\right)} & \leq \sup _{0<\delta<1} \int_{\Omega_{T}}\left|\theta_{\delta}-T_{k}\left(\theta_{\delta}\right)\right| d x d t \leq C \sup _{0<\delta<1} \int_{\left\{\theta_{\delta}>k\right\}} \theta_{\delta} d x d t \\
& \leq \frac{C}{k} \sup _{0<\delta<1} \int_{\left\{\theta_{\delta}>k\right\}} \theta_{\delta}^{2} d x d t \leq \frac{C}{k} .
\end{aligned}
$$

We infer from (49) that for any $k \geq 1$,

$$
\left\|\frac{\overline{\rho_{\delta} \theta_{\delta}}-\rho \theta}{1+\rho}\right\|_{L^{1}\left(\Omega_{T}\right)} \leq \frac{C}{k}
$$

which implies, in the limit $k \rightarrow \infty$, that

$$
\overline{\rho_{\delta} \theta_{\delta}}=\rho \theta \quad \text { a.e. in } \Omega_{T} .
$$

Step 4: Pointwise convergence of $\left(\theta_{\delta}\right)$. We prove via the Aubin-Lions lemma that $E_{\delta}=\theta_{\delta}+\frac{3}{2} \rho_{\delta} \theta_{\delta}$ is strongly convergent. We know from Lemma 4 that $\left(E_{\delta}\right)$ is bounded in $L^{1}\left(0, T ; W^{1,1}(\Omega)\right)$. For the time derivative of $E_{\delta}$, we estimate (44) for $\psi_{2} \in C_{0}^{\infty}\left(\Omega_{T}\right)$ :

$$
\begin{aligned}
\left|\int_{0}^{T}\left\langle\partial_{t} E_{\delta}, \psi_{2}\right\rangle d t\right| \leq & \left|\int_{0}^{T} \int_{\Omega}\left(\theta_{\delta}+\frac{5}{2} \rho_{\delta} \theta_{\delta}^{2}+\frac{\delta}{3} \theta_{\delta}^{3}\right) \Delta \psi_{2} d x d t\right| \\
& +\left|\delta \int_{0}^{T} \int_{\Omega} \theta_{\delta}^{-N} \log \left(\theta_{\delta}\right) \psi_{2} d x d t\right| \\
\leq & C\left(\left\|\theta_{\delta}\right\|_{L^{2}\left(\Omega_{T}\right)}+\left\|\rho_{\delta} \theta_{\delta}^{2}\right\|_{L^{3 / 2}\left(\Omega_{T}\right)}\right. \\
& \left.+\delta\left\|\theta_{\delta}^{3}\right\|_{L^{4 / 3}\left(\Omega_{T}\right)}\right)\left\|\Delta \psi_{2}\right\|_{L^{4}\left(\Omega_{T}\right)} \\
& +C\left(1+\delta\left\|\theta_{\delta}^{-N} \log \theta_{\delta}\right\|_{L^{6 / 5}\left(\Omega_{T}\right)}\right)\left\|\psi_{2}\right\|_{L^{6}\left(\Omega_{T}\right)}
\end{aligned}
$$

Taking into account estimate (39) and again using Lemma 3, we infer that

$$
\left\|\partial_{t} E_{\delta}\right\|_{L^{6 / 5}\left(0, T ; W^{2,4}(\Omega)^{\prime}\right)} \leq C
$$

We apply the Aubin-Lions lemma to $\left(E_{\delta}\right)$ to obtain the existence of a subsequence which is not relabeled such that, as $\delta \rightarrow 0,\left(E_{\delta}\right)$ converges strongly in $L^{\eta}\left(\Omega_{T}\right)$ for $\eta<2$. Since $\left(1+\theta_{\delta}\right)^{-1}$ converges weakly in $L^{\eta}\left(\Omega_{T}\right)$ for any $\eta<\infty$, we find that

$$
\overline{\left(\theta_{\delta}+\frac{3}{2} \rho_{\delta} \theta_{\delta}\right)\left(1+\theta_{\delta}\right)^{-1}}=\overline{\left(\theta_{\delta}+\frac{3}{2} \rho_{\delta} \theta_{\delta}\right)} \overline{\left(1+\theta_{\delta}\right)^{-1}} \quad \text { a.e. in } \Omega_{T} .
$$

We choose $g(s)=s(1+s)^{-1}$ in (48) and recall (50):

$$
\overline{\rho_{\delta} \theta_{\delta}\left(1+\theta_{\delta}\right)^{-1}}=\rho \overline{\theta_{\delta}\left(1+\theta_{\delta}\right)^{-1}}, \quad \overline{\rho_{\delta} \theta_{\delta}}=\rho \theta \quad \text { a.e. in } \Omega_{T} .
$$


Using these expressions, we deduce from (51) that

$$
\begin{aligned}
\left(1+\frac{3}{2} \rho\right) & \overline{\theta_{\delta}\left(1+\theta_{\delta}\right)^{-1}}=\overline{\theta_{\delta}\left(1+\theta_{\delta}\right)^{-1}+\frac{3}{2} \rho_{\delta} \theta_{\delta}\left(1+\theta_{\delta}\right)^{-1}} \\
& =\overline{\left(\theta_{\delta}+\frac{3}{2} \rho_{\delta} \theta_{\delta}\right)} \overline{\left(1+\theta_{\delta}\right)^{-1}}=\overline{\theta_{\delta}} \overline{\left(1+\theta_{\delta}\right)^{-1}}+\frac{3}{2} \overline{\rho_{\delta} \theta_{\delta}} \overline{\left(1+\theta_{\delta}\right)^{-1}} \\
& =\left(1+\frac{3}{2} \rho\right) \theta \overline{\left(1+\theta_{\delta}\right)^{-1}} \quad \text { a.e. in } \Omega_{T} .
\end{aligned}
$$

This means that

$$
\overline{\theta_{\theta}\left(1+\theta_{\delta}\right)^{-1}}=\theta \overline{\left(1+\theta_{\delta}\right)^{-1}} \text { a.e. in } \Omega_{T} .
$$

We apply [13, Theorem 10.19] to the strictly decreasing function $s \mapsto(1+s)^{-1}$ for $s \geq 0$ to conclude that

$$
\overline{\left(1+\theta_{\delta}\right)^{-1}}=(1+\theta)^{-1} \quad \text { a.e. in } \Omega_{T} .
$$

The strict convexity of $s \mapsto(1+s)^{-1}$ then implies, by [13, Theorem 10.20], that $\theta_{\delta} \rightarrow \theta$ a.e. in $\Omega_{T}$. We deduce from the $L^{2}\left(\Omega_{T}\right)$ bound for $\left(\theta_{\delta}\right)$ from Lemma 3 that this convergence is in fact strong in $L^{1}\left(\Omega_{T}\right)$.

Step 5: Limit $\delta \rightarrow 0$ in equations (43)-(44). We know from (42) that $\left(\nabla\left(\rho_{\delta} \theta_{\delta}\right)\right)$ is bounded in $L^{4 / 3}\left(\Omega_{T}\right)$. Thus, up to a subsequence, $\nabla\left(\rho_{\delta} \theta_{\delta}\right) \rightarrow$ $\zeta_{1}$ weakly in $L^{4 / 3}\left(\Omega_{T}\right)$ for some $\zeta_{1} \in L^{4 / 3}\left(\Omega_{T}\right)$. Since $\rho_{\delta} \theta_{\delta} \rightarrow \rho \theta$ weakly in $L^{1}\left(\Omega_{T}\right)$, by $(50)$, we infer that $\zeta_{1}=\nabla(\rho \theta)$, i.e.

$$
\nabla\left(\rho_{\delta} \theta_{\delta}\right) \rightarrow \nabla(\rho \theta) \quad \text { weakly in } L^{4 / 3}\left(\Omega_{T}\right) .
$$

We know from Lemma 3 that $\left(\rho_{\delta} \theta_{\delta}^{2}\right)$ is bounded in $L^{3 / 2}\left(\Omega_{T}\right)$, so that up to a subsequence, $\rho_{\delta} \theta_{\delta}^{2} \rightarrow \zeta_{2}$ weakly in $L^{3 / 2}\left(\Omega_{T}\right)$. We deduce from the strong convergence of $\left(\theta_{\delta}\right)$ and the boundedness of $s \mapsto\left(1+s^{2}\right)^{-1}$ that $\left(1+\theta_{\delta}^{2}\right)^{-1} \rightarrow$ $\left(1+\theta^{2}\right)^{-1}$ strongly in $L^{\eta}\left(\Omega_{T}\right)$ for any $\eta<\infty$. Therefore,

$$
\frac{\rho_{\delta} \theta_{\delta}^{2}}{1+\theta_{\delta}^{2}} \rightarrow \frac{\zeta_{2}}{1+\theta^{2}} \quad \text { weakly in } L^{1}\left(\Omega_{T}\right) .
$$

An application of (48) with $g(s)=s^{2}\left(1+s^{2}\right)^{-1}$ together with the strong convergence of $\left(\theta_{\delta}\right)$ leads to

$$
\frac{\rho_{\delta} \theta_{\delta}^{2}}{1+\theta_{\delta}^{2}} \rightarrow \frac{\rho \theta^{2}}{1+\theta^{2}} \quad \text { weakly in } L^{1}\left(\Omega_{T}\right) .
$$

Hence, $\zeta_{2}=\rho \theta^{2}$ a.e. in $\Omega_{T}$ and

$$
\rho_{\delta} \theta_{\delta}^{2} \rightarrow \rho \theta^{2} \quad \text { weakly in } L^{3 / 2}\left(\Omega_{T}\right) .
$$

Furthermore, it follows from (33), Lemma 3, and (39) that

$$
\begin{aligned}
\delta \phi_{\delta} \rightarrow 0 & \text { strongly in } L^{2}\left(0, T ; H^{1}(\Omega)\right), \\
\delta \theta_{\delta}^{3} \rightarrow 0 & \text { strongly in } L^{4 / 3}\left(\Omega_{T}\right), \\
\delta \theta_{\delta}^{-N} \log \theta_{\delta} \rightarrow 0 & \text { strongly in } L^{6 / 5}\left(\Omega_{T}\right) .
\end{aligned}
$$


For any $\psi_{1} \in L^{4}\left(0, T ; W^{1,4}(\Omega)\right)$, we have

$$
\begin{aligned}
\left|\int_{0}^{T}\left\langle\partial_{t} \rho_{\delta}, \psi_{1}\right\rangle d t\right| \leq & \frac{3}{2}\left\|\nabla\left(\rho_{\delta} \theta_{\delta}\right)\right\|_{L^{4 / 3}\left(\Omega_{T}\right)}\left\|\nabla \psi_{1}\right\|_{L^{4}\left(\Omega_{T}\right)} \\
& +\delta\left\|\phi_{\delta}\right\|_{L^{2}\left(0, T ; H^{1}(\Omega)\right)}\left\|\psi_{1}\right\|_{L^{2}\left(0, T ; H^{1}(\Omega)\right)} \leq C .
\end{aligned}
$$

Hence, up to subsequences,

$$
\begin{aligned}
\partial_{t} \rho_{\delta} \rightarrow \partial_{t} \rho & \text { weakly in } L^{4 / 3}\left(0, T ; W^{1,4}(\Omega)^{\prime}\right), \\
\partial_{t} E_{\delta} \rightarrow \partial_{t} E & \text { weakly in } L^{6 / 5}\left(0, T ; W^{2,4}(\Omega)^{\prime}\right) .
\end{aligned}
$$

We deduce from the bound for $\left(\log \theta_{\delta}\right)$ in $L^{\infty}\left(0, T ; L^{1}(\Omega)\right)$ that $\theta>0$ a.e. in $\Omega_{T}$.

We claim that $\left(\rho_{\delta}\right)$ also converges strongly. Indeed, the a.e. convergence of $\left(E_{\delta}\right)$ and $\left(\theta_{\delta}\right)$ imply that $\rho_{\delta}=\frac{2}{3}\left(E_{\delta} / \theta_{\delta}-1\right) \rightarrow \rho$ a.e. in $\Omega_{T}$. The $L^{\infty}\left(0, T ; L^{1}(\Omega)\right)$ bound for $\left(\rho_{\delta} \log \rho_{\delta}\right)$ from $(30)$ shows that $\left(\rho_{\delta}\right)$ is equi-integrable, and together with its a.e. convergence, we conclude from the de la Vallée-Poussin theorem [10, Chap. 8, Sect. 1.7, Corollary 1.3] that

$$
\rho_{\delta} \rightarrow \rho \quad \text { strongly in } L^{1}\left(\Omega_{T}\right) \text {. }
$$

The positivity of $\rho_{\delta}$ implies that $\rho \geq 0$ a.e. in $\Omega_{T}$. Note, however, that we cannot conclude that $\rho>0$ a.e., since the control on $\phi_{\delta}$ is now lost.

Convergences (52)-(55) allow us to perform the limit $\delta \rightarrow 0$ in (43)-(44) showing that $(\rho, \theta)$ solves $(9)-(10)$. Theorem 1 is proved.

Funding Open access funding provided by Austrian Science Fund (FWF).

Open Access. This article is licensed under a Creative Commons Attribution 4.0 International License, which permits use, sharing, adaptation, distribution and reproduction in any medium or format, as long as you give appropriate credit to the original author(s) and the source, provide a link to the Creative Commons licence, and indicate if changes were made. The images or other third party material in this article are included in the article's Creative Commons licence, unless indicated otherwise in a credit line to the material. If material is not included in the article's Creative Commons licence and your intended use is not permitted by statutory regulation or exceeds the permitted use, you will need to obtain permission directly from the copyright holder. To view a copy of this licence, visit http://creativecommons. org/licenses/by/4.0/.

Publisher's Note Springer Nature remains neutral with regard to jurisdictional claims in published maps and institutional affiliations.

\section{References}

[1] Abels, H., Depner, D., Garcke, H.: On an incompressible Navier-Stokes/CahnHilliard system with degenerate mobility. Ann. Inst. H. Poincaré Anal. Non Lin. 30, 1175-1190 (2013) 
[2] Alí, G., Romano, V.: Existence and uniqueness for a two-temperature energytransport model for semiconductors. J. Math. Anal. Appl. 449, 1248-1264 (2017)

[3] Allegretto, W., Xie, H.: Nonisothermal semiconductor systems. In: Liu, X., Siegel, D. (eds.) Comparison Methods and Stability Theory. Lecture Notes Pure and Applied Mathematics, vol. 162, pp. 17-24. Marcel Dekker, New York (1994)

[4] Biler, P., Nadzieja, T.: Structure of steady states for Streater's energy-transport models of gravitating particles. Topol. Methods Nonlinear Anal. 19, 283-301 (2002)

[5] Braukhoff, M., Jüngel, A.: Energy-transport systems for optical lattices: derivation, analysis, simulation. Math. Models Methods Appl. Sci. 28, 579-614 (2018)

[6] Chen, L., Hsiao, L.: The solution of Lyumkis energy transport model in semiconductor science. Math. Methods Appl. Sci. 26, 1421-1433 (2003)

[7] Chen, X., Jüngel, A., Liu, J.-G.: A note on Aubin-Lions-Dubinskiǔ lemmas. Acta Appl. Math. 133, 33-43 (2014)

[8] Degond, P., Génieys, S., Jüngel, A.: A system of parabolic equations in nonequilibrium thermodynamics including thermal and electrical effects. J. Math. Pures Appl. 76, 991-1015 (1997)

[9] Dreher, M., Jüngel, A.: Compact families of piecewise constant functions in $L^{p}(0, T ; B)$. Nonlinear Anal. 75, 3072-3077 (2012)

[10] Ekeland, I., Temam, R.: Convex Analysis and Variational Problems. NorthHolland, Amsterdam (1976)

[11] Elliott, C., Garcke, H.: On the Cahn-Hilliard equation with degenerate mobility. SIAM J. Math. Anal. 27, 404-423 (2000)

[12] Favre, G., Pirner, M., Schmeiser, C.: Thermalization of a rarefied gas with total energy conservation: existence, hypocoercivity, macroscopic limit. Submitted for publication (2020). arXiv:2012.07503

[13] Feireisl, E., Novotný, A.: Singular Limits in Thermodynamics of Viscous Flows. Birkhäuser, Basel (2009)

[14] Jüngel, A.: Transport Equations for Semiconductors. Lecture Notes in Physics, vol. 773. Springer, Berlin (2009)

[15] Jüngel, A., Pinnau, R., Röhrig, E.: Existence analysis for a simplified transient energy-transport model for semiconductors. Math. Methods Appl. Sci. 36, 1701$1712(2013)$

[16] Roubíček, T.: Nonlinear Partial Differential Equations with Applications. Birkhäuser, Basel (2005)

[17] Stratton, R.: Diffusion of hot and cold electrons in semiconductor barriers. Phys. Rev. 126, 2002-2014 (1962)

[18] Temam, R.: Infinite-Dimensional Dynamical Systems in Mechanics and Physics, 2nd edn. Springer, New York (1997) 
[19] Zamponi, N., Jüngel, A.: Global existence analysis for degenerate energytransport models for semiconductors. J. Differ. Equ. 258, 2339-2363 (2015)

Gianluca Favre and Christian Schmeiser

Fakultät für Mathematik

Universität Wien

Oskar-Morgenstern-Platz 1

1090 Vienna

Austria

e-mail: gianluca.favre@univie.ac.at

Christian Schmeiser

e-mail: christian.schmeiser@univie.ac.at

Ansgar Jüngel

Institute for Analysis and Scientific Computing

Vienna University of Technology

Wiedner Hauptstraß e 8-10

1040 Vienna

Austria

e-mail: juengel@tuwien.ac.at

Nicola Zamponi

School of Business Informatics and Mathematics

University of Mannheim

B6, 28

68159 Mannheim

Germany

e-mail: nzamponi@mail.uni-mannheim.de

Received: 13 August 2020.

Accepted: 28 April 2021. 